\title{
Chromatin Compaction, Auxeticity, and the Epigenetic Landscape of Stem Cells
}

\author{
Kamal Tripathi ${ }^{*}$ and Gautam I. Menon $\odot^{\dagger}$ \\ The Institute of Mathematical Sciences, C.I.T. Campus, Taramani, Chennai 600 013, India \\ and Homi Bhabha National Institute, Training School Complex, Anushaktinagar, Mumbai 400 094, India
}

(Received 27 April 2019; revised manuscript received 3 August 2019; published 28 October 2019)

\begin{abstract}
When embryonic stem cells differentiate, the mechanical properties of their nuclei evolve en route to their terminal state. Measurements of the deformability of cell nuclei in the transitional state that intervenes between the embryonic stem-cell state and the differentiation primed state of mouse stem cells indicate that such nuclei are auxetic; i.e., they have a negative Poisson's ratio. We show, using a theoretical model, how this remarkable mechanical behavior results from the coupling between chromatin compaction states and nuclear shape. Our biophysical approach, which treats chromatin as an active polymer system whose mechanics is modulated by nucleosome binding and unbinding, reproduces experimental results. It provides testable predictions for changes in chromatin compaction as a function of applied force, for the correlations of chromatin compaction and nuclear shape, and for the in-phase and out-of-phase response of these quantities to an applied uniaxial oscillatory force. Our model yields a biophysical interpretation of the epigenetic landscape of stem cells, also suggesting how this landscape might be probed experimentally.
\end{abstract}

\section{INTRODUCTION}

Embryonic stem cells (ES cells) occupy the apex of a hierarchy of cellular states [1]. They can self-renew, maintaining their "stemness," but also differentiate into varied cellular lineages when supplied with appropriate biochemical or mechanical cues. This is the property of pluripotency $[2,3]$. Cell lineage choice results from shifts in patterns of gene expression, controlled by the rewiring of gene regulatory networks. Such rewiring can arise because the transcription factors that control the production of RNA are present at different levels in different cell types. But such shifts can also occur through biochemical changes at the DNA level, such as the methylation of cytosine residues, as well as through modifications in histone and other architectural proteins that bind DNA [4,5]. Such epigenetic modifications, thus termed because they do not affect DNA sequence, alter the local structure and biophysical properties of chromatin, the term applied to DNA in its natural state in the nuclei of living cells.

\footnotetext{
*kamalt@imsc.res.in

†menon@imsc.res.in

*Present address: Department of Physics, Ashoka University, Plot No. 2, Rajiv Gandhi Education City, National Capital Region, P.O. Rai, Sonepat, Haryana 131 029, India.

Published by the American Physical Society under the terms of the Creative Commons Attribution 4.0 International license. Further distribution of this work must maintain attribution to the author(s) and the published article's title, journal citation, and DOI.
}

Changes in patterns of gene expression should have biophysical correlates since they require actively transcribed genes to be more accessible than silenced genes [6]. Regions of chromatin that see higher levels of transcriptional activity are typically more loosely packed (euchromatin) than gene-poor and relatively more compact (heterochromatin) regions. These regions can be identified through local epigenetic marks [7]. The local state of chromatin compaction is clearly relevant to the biophysics of chromatin. It may assume added importance in the highly dynamic stem-cell state since ES cells are known to be transcriptionally hyperactive $[8,9]$. In addition, ES cell chromatin is "hyperdynamic," with histones binding and unbinding locally at an enhanced rate compared to differentiated cells [10]. This increased rate of binding and unbinding leads to larger fluctuations in chromatin packaging. Stem-cell chromatin is thus more fluidlike than the chromatin of differentiated cells. Such fluidity likely contributes to the maintenance of pluripotency [11].

Before lineage commitment, ES cells exhibit decondensed chromatin and soft nuclei. A slowing down of histone dynamics, a stiffening of the nuclear envelope, and global chromatin remodeling all accompany differentiation, resulting in a transition from a more open chromatin configuration to a more compact state [12-16]. The interplay of chromatin packaging with fluctuations of the relatively pliable chromatin-enclosing nuclear envelope might reasonably be expected to underly the special biophysical properties of the stem-cell nucleus $[17,18]$. Purely mechanical cues, such as substrate stiffness or substrate structure, are sufficient 
to drive stem-cell differentiation into preferred lineages suggesting a biophysical link between nuclear mechanics, chromatin packaging, and lineage choice [12,19,20].

Waddington originally visualized the differentiation of stem cells in terms of a set of branching tracks representing different cell fate choices [21]. A subsequent, more pictorial version of this idea used the analogy of a ball rolling along an "epigenetic landscape" with minima chosen to represent stable differentiated states [22,23]. Stable positions in this landscape have been argued to correspond to attractors of a highdimensional nonlinear dynamical system controlled by feedback [24]. This provides a particularly appealing and pictorial way of understanding how stem-cell differentiation into specific cell lineages can be visualized. Such ideas naturally connect to other landscape descriptions of biophysical states and phenomena $[25,26]$. However, the experimental corollaries of an epigenetic landscape and how, in particular, nuclear mechanics might enter its description are not well understood.

We ask whether recent biophysical measurements of the mechanical properties of stem-cell nuclei can provide insights into these broader questions [27-29]. We first note that almost all materials have a positive Poisson's ratio, becoming wider in the transverse direction when compressed uniaxially along a longitudinal dimension [30,31]. Materials with a negative Poisson's ratio, such as foams, are termed auxetics [32,33]. Pagliara et al. [34] report results from atomic force microscopy (AFM) measurements of the reduced modulus $K=E /\left(1-\nu^{2}\right)$, with $E$ the uniaxial stiffness and $\nu$ the Poisson's ratio, of naive mouse embryonic stem-cell (N-ESC) nuclei exiting the pluripotent state en route to differentiation via a transitional state (T-ESC) and a differentiation primed $(\mathrm{P})$ state. In the transitional state, obtained when specific inhibitors preventing the transition to a differentiation primed state are removed, the cell nuclei become smaller by about $5 \%-10 \%$ in cross section when compressed to the level of about $2 \mu \mathrm{m}$ with the AFM probe [34]. Similar results were obtained by observing changes in nuclear dimensions when cells in the T-ESC state were set in flow along a microchannel. Whereas both the N-ESC and the P states exhibit a positive Poisson's ratio, the T-ESC state that intervenes between them is thus auxetic, with a negative Poisson's ratio. Pagliara et al. suggest that the auxetic phenotype might be connected to chromatin decondensation since chromatin in the transitional state is less condensed than in either the embryonic stem-cell state or the differentiation primed state [34]. Disrupting the actin cytoskeleton through Cytochalasin D treatment did not remove auxeticity, indicating that it might naturally originate in the biophysical properties of the nucleus itself and not of the extranuclear environment.
The model we describe here addresses these experiments, placing them in a wider context of our understanding of the epigenetic landscape of stem cells. It uses four biophysical assumptions. These follow from the experimental observations. First, the nucleus in the auxetic regime is compressible, a fundamental property of the auxetic state $[35,36]$. Second, mechanical response to a uniaxial external force in such a regime must distinguish between directions parallel to the force and perpendicular to it, although such anisotropy need not be intrinsic to isolated stem cells in the absence of an applied force [37]. Third, a number of experiments indicating chromatin fluidity in all but terminally differentiated states argue that chromatin is best described as a confined, active polymer fluid in a semidilute regime [38]. (Indeed, the formation of heterochromatin foci has been discussed in analogy with active phase separation in liquidliquid mixtures $[39,40]$.) An alternative view of auxeticity, which considers the nucleoplasm to be a gel and uses ideas from phase separation, is described in Ref. [41]. We treat activity as equivalent to a (higher) effective temperature [42-44]. Fourth and finally, we assume that auxetic behavior arises from the form of the coupling of chromatin compaction states to mechanical variables, which we choose as nuclear dimensions parallel to, as well as perpendicular to, the applied force. These four assumptions, all reasonable from a biophysical standpoint, inform our mathematical model. We use them to derive a model nonlinear dynamical system describing auxetic behavior in the transitional state of stem cells.

\section{MATERIALS AND METHODS}

We first identify relevant variables of interest, in particular, those that are amenable to measurement. Figure 1(a) shows a schematic of the experiments of Ref. [34], while Fig. 1(b) illustrates how the on-off dynamics of nucleosomes in the stem-cell state might alter chromatin packaging. Figure 1(c) illustrates the definitions of the fundamental mechanical variables that enter our model. We use a single variable, labeled $\Psi$, to describe nucleosome-induced compaction of chromatin. The variable $\Psi$ can be thought of as representing the number of nucleosomes bound to chromatin at a given time, with the biophysical interpretation that a larger number of bound nucleosomes yields a more compact chromatin structure. The structural variables $R_{\|}=R_{0}+\delta R_{\|}$ and $R_{\perp}=R_{0}+\delta R_{\perp}$ denote nuclear dimensions parallel and perpendicular to the direction of the applied force $f$, as shown. Figures 1(d)-1(e) illustrate how nuclei deform under force in both the nonauxetic (d) and the auxetic (e) cases. Finally, Figs. 1(f) and 1(g) supply schematics of auxetic and nonauxetic response to a force $f$, in the variables $\Psi, R_{\|}$, and $R_{\perp}$. How to derive the schematics of Figs. 1(f) and $1(\mathrm{~g})$, including the behavior of $\Psi$ in both the auxetic and nonauxetic cases predicted by the theoretical formulation, is the subject of this paper. 

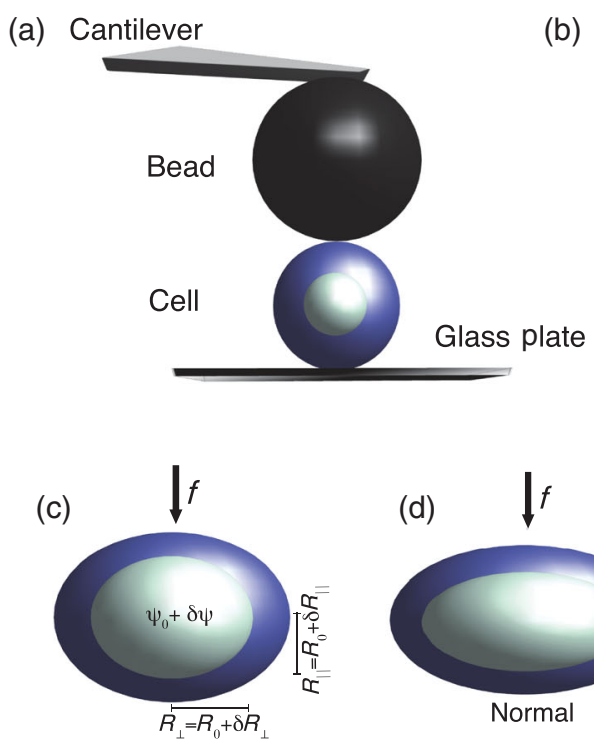

(b)

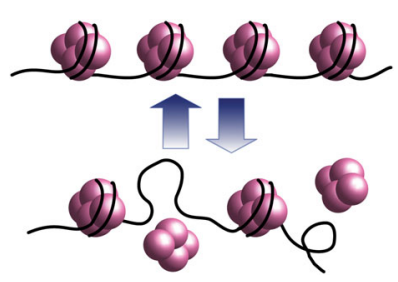

(f)

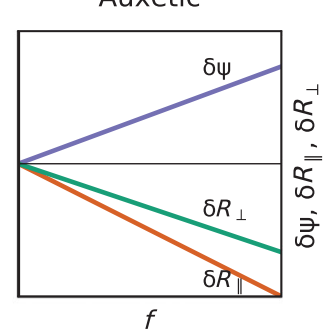

(g)

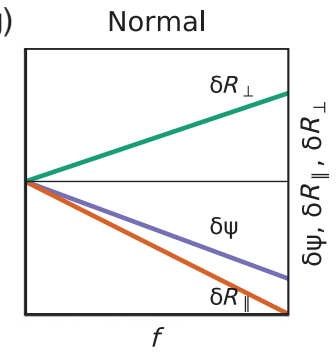

FIG. 1. (a) Schematic of the AFM experiment of Ref. [34]. (b) Fluctuations in chromatin compaction arising from the fast on-off dynamics of nucleosomes in the stem-cell state, where histones are hyperdynamic. (c) Definitions of the variables $\Psi, R_{\|}$, and $R_{\perp}$ in the AFM-based indentation experiment, including the applied force $f$ arising from the indentation. (d) Illustration of normal, i.e., nonauxetic behavior in the experiments, showing how the nucleus expands in the direction perpendicular to the applied force $f$, while the nuclear dimension in the direction parallel to the force contracts. (e) Illustration of auxetic behavior, showing how the nucleus contracts both in the direction perpendicular to the applied force and in the direction parallel to it. The schematic plots in (f) for the nonauxetic case and (g) for the auxetic case show how the variables $\Psi, R_{\|}$, and $R_{\perp}$ behave in both limits as $f$ is increased from zero. The unperturbed nucleus is taken to be spherical.

\section{A. Model description}

Our equations are formulated in terms of $\delta \Psi, \delta R_{\|}$, and $\delta R_{\perp}$ defined as in Fig. 1 and derived below. The equations describing how these quantities change in time take the form

$$
\begin{aligned}
& \frac{d(\delta \Psi)}{d t}=-\frac{d V(\delta \Psi)}{d \delta \Psi}+B\left(\frac{1}{2} \delta R_{\|}+\delta R_{\perp}\right)+\eta_{\Psi}, \\
& \frac{d\left(\delta R_{\|}\right)}{d t}=-C \delta R_{\|}-D \delta \Psi-f_{\|}+\eta_{R}, \\
& \frac{d\left(\delta R_{\perp}\right)}{d t}=-C \delta R_{\perp}-D \delta \Psi+f_{\perp}+\eta_{R}^{\prime} .
\end{aligned}
$$

We have used our freedom to choose units suitably to "de-dimensionalize" the coefficients that appear in these equations. The first term on the right-hand side of each of these equations represents the independent relaxation of fluctuations away from $\left\{\Psi_{0}, R_{0}\right\}$. We assume that the $\delta \Psi$ variable relaxes subject to an effective potential $V(\delta \Psi)$. The interpretation of this term will become clearer as we proceed. The second term couples $\delta \Psi$ to the mechanical variables $R_{\|}=R_{0}+\delta R_{\|}$and $R_{\perp}=R_{0}+\delta R_{\perp}$, with coefficient $B$; the relative factor of 2 accounts for the $3 \mathrm{D}$ geometry. This coupling appears to lowest order in the fluctuations $\delta R_{\|}$and $\delta R_{\perp}$. This is the simplest form that these equations could take. Their biophysical content lies in the estimates of the numerical values associated with the coefficients. More subtly, the coupling between chromatin compaction and nuclear dimensions is to be found in the cross terms in Eq. (1).

In the absence of a force, $R_{\|}$and $R_{\perp}$ are equivalent. The symmetry between them is broken only by $f_{\|}$and $f_{\perp}$. These forces represent both external forces and forces that arise from the remodeling of the extranuclear actin cytoskeleton, which can be assumed to be uniform if $f_{\|}=0$. We can assume that $f_{\|}$couples primarily to $\delta R_{\|}$, whereas $f_{\perp}$ couples to $\delta R_{\perp}$. In the absence of external forces, the two equations reduce to a single one. The quantity $C$ represents a ratio of timescales for the relaxation of the $\Psi$ and the $R$ variables. If $\Psi_{0}$ represents a stable state, or at least a state that evolves slowly on the timescale of the fluctuations $\delta \Psi$, we can expand in these fluctuations. At the simplest level then, these fluctuations are subject to a harmonic potential. The case where $\delta R_{\|}=\delta R_{\perp} \equiv \delta R$, with $f_{\perp}=f_{\|}=0$ and the $V(\delta \Psi)$ term chosen to be bistable, was studied in Ref. [45], in the context of nuclear-size oscillations in the ES state of mouse stem cells. We use this more specific form of these equations when we identify signals of auxetic behavior in fluctuations within the undeformed steady state. Our results suggest that signatures of the transition between auxetic and nonauxetic behavior might be most easily seen in these fluctuations.

In Ref. [45], in a description of enhanced fluctuations in mouse N-ESCs, $B>0$ was assumed. The physical 
interpretation in that work was that increasing the size of the nucleus would expose binding sites for histones. This case leads to a concomitant increase in $\Psi$, which would then cause the nucleus to shrink [45]. The coupled dynamics of the fast histone on-off rates in the hyperdynamic case with the slower fluctuations in nuclear size leads to interesting fluctuation behavior. Such a choice of sign inevitably leads to nonauxetic behavior; see below.

Experiments show that chromatin is most decondensed in the transitional state, as opposed to either the ES state or the differentiation primed states between which it intervenes [34]. A further expansion of nuclear dimensions might then be expected to result in the expulsion of nucleosomes, rather than their accumulation, in this intermediate state. Incorporating this idea into the modeling requires that we consider the case where $B<0$. Indeed, treating the naive pluripotent ESC state with trichostatin A, a Histone deacetylase (HDAC) inhibitor that globally decondenses chromatin makes the N-ESC auxetic, arguing for the connection to our modeling. We can thus view the transition between the naive stem cell, the transitional state, and the differentiation primed state in terms of a reentrant behavior in the sign of $B$. This prediction is experimentally testable.

The on-off dynamics of histones is inherently noisy. Our equations account for such stochastic effects, represented as additive noise with standard properties, with terms represented by $\eta_{\Psi}, \eta_{R}$, and $\eta_{R}^{\prime}$. In general, the effects of the noise should be most significant for the fast fluctuating $\Psi$ variable. We thus choose to retain only the Gaussiandistributed, delta-correlated $\eta_{\Psi}$ term in our equations, setting $\eta_{R}=\eta_{R}^{\prime}=0$.

\section{B. Derivation of model equations: $\boldsymbol{R}$ equation}

Our equations are motivated in the following way, illustrated, for simplicity, in the isotropic case: Assume first that the nucleus is a sphere of radius $R$, prestressed by chromatin polymer pressure. Given compressibility, assume that the dominant modes of fluctuations are breathing modes, associated with an elastic energy $E_{\mathrm{el}}=\left[K_{a} /\left(2 a_{0}\right)\right] \times$ $\left[\left(a-a_{0}\right)^{2}\right]$, which penalizes changes in area $a$ from an unstressed or even prestressed state where the area is $a_{0}$. This term also accounts for the contribution of the actin cytoskeleton, which enters as a modified area expansion modulus $K_{a}$. Fluid flow in and out of the sphere, driven by a pressure imbalance, leads to volume changes and is resisted by a cost for deviations in the surface area from its preferred value.

Describing stem-cell chromatin as a polymer solution at an effective (active) temperature $T^{*}$, the free energy of the polymer solution in units of $k_{B} T^{*}$ is of the form $f_{m}(\phi)=$ $(\phi / N) \ln \phi+(1-\phi) \ln (1-\phi)+\chi \phi(1-\phi)+1 /\left(24 \pi \xi^{3}\right)$, where $\phi$ is the volume fraction of the polymer and $\xi \sim$ $\phi^{-\nu /(3 \nu-1)}$ is the correlation term arising from monomer density fluctuations [46,47]. Activity enters as an effective temperature $T^{*}$. More subtly, it modifies the effective Flory term $\chi$.
The polymer osmotic pressure follows from $\Pi=$ $\left[\left(k_{B} T^{*}\right) / \nu_{c}\right]\left[\phi\left(\partial f_{m} / \partial \phi\right)-f_{m}\right]$, which yields $\Pi=$ $\left[\left(k_{B} T^{*}\right) / \nu_{c}\right]\left[(\phi / N)-\ln (1-\phi)-\phi-\chi \phi^{2}\right]$, where $k_{B}$ is the Boltzmann constant, $T^{*}$ is the effective temperature, $\nu_{c}$ is the monomer volume, $\phi$ is the volume fraction of the polymer, and $N$ is the degree of polymerization [46]. Physically, $\chi$ alters the relative balance of chromatinchromatin and chromatin-solvent interactions, as manifest in the compaction state of chromatin. The effective Flory parameter $\chi$ is then tuned by the fraction of bound nucleosomes, which controls $\Psi: \chi=\chi(\Psi)$. We then have

$$
\Delta \Pi=-\frac{k_{B} T^{*} \phi_{0}^{2}}{\nu_{c}} \chi^{\prime} \delta \Psi
$$

where $\chi^{\prime}=d \chi / d \Psi$. Penalizing fluctuations of the nuclear envelope from its preferred area $a_{0}$ yields a restoring force of the form $F=-16 \pi K_{a} \delta R$ and thus a pressure term

$$
\Delta P=\frac{4 K_{a}}{R_{0}^{2}} \delta R
$$

Darcy's law provides an expression for the rate of change of volume $d V / d t=[(\kappa A) /(\mu L)](\Delta \Pi-\Delta P)$, where $\kappa$ is the permeability $\left(\mathrm{m}^{2}\right), A$ is the area of the nucleus, $\mu$ the viscosity, and $L$ the length over which the pressure drops [48]. This expression yields, where we use the notation $\dot{R}=d R / d t, \dot{\Psi}=d \Psi / d t$,

$$
\begin{aligned}
\dot{R} & =-\left(\frac{4 \kappa K_{a}}{\mu L R_{0}^{2}}\right) \delta R-\left(\frac{\kappa k_{B} T^{*} \phi_{0}^{2}}{\mu L \nu_{c}} \chi^{\prime}\right) \delta \Psi, \\
& \Rightarrow \dot{R}=-C \delta R-D \delta \Psi
\end{aligned}
$$

where $C=\left[\left(4 \kappa K_{a}\right) /\left(\mu L R_{0}^{2}\right)\right]$ and $D=\left[\left(\kappa k_{B} T^{*} \phi_{0}^{2}\right) /\right.$ $\left.\left(\mu L \nu_{c}\right)\right] \chi^{\prime}$. Note that $D>0$ is required by the biophysical input that the binding of histones must lead to a contraction in DNA. The larger the polymer-solvent interaction, the smaller the Flory-Huggins $\chi$ parameter, implying that we can interpret histone binding and the consequent compaction of DNA as an effective decrease of the polymer-solvent interaction with histone binding. This result then implies that the effective Flory-Huggins parameter should increase with $\Psi$, implying that $\chi^{\prime}>0$. Here, $\Delta \Pi-\Delta P$ provides the driving force, in this case, the difference between polymer and Laplace pressures relative to their unperturbed values. This case holds in the absence of a force $f$.

This result is easily generalized to the anisotropic case.

\section{Derivation of model equations: $\Psi$ equation}

We now discuss the dynamics of $\delta \Psi$. First, ignoring the coupling to $R_{\perp}$ and $R_{\|}$, we model fluctuations in $\Psi$ as relaxing in an overdamped manner to $\Psi_{0}$. This dynamics explores the one-dimensional landscape defined through 
the effective potential $V(\delta \Psi)$, with $\Psi_{0}$ at least a local minimum. Consider $N$ nucleosome binding sites on a piece of DNA, in equilibrium with unbound nucleosomes at chemical potential $\mu$, with the energy gain from nucleosome binding to DNA being $\epsilon$. The probability of the nucleosome being bound is the Fermi function $p=$ $1 /\left(1+e^{(\epsilon-\mu) / k_{B} T}\right)$ since this is an effective two-state problem at each binding site. If the radius of the confining sphere is changed from $R_{0}$ to $R=R_{0}+\delta R$, the DNA will stretch in place, altering $\epsilon$. Assuming $\epsilon=\epsilon(R), \epsilon(R)=$ $\epsilon\left(R_{0}+\delta R\right) \approx \epsilon\left(R_{0}\right)+\epsilon^{\prime} \delta R$, where $\epsilon^{\prime}=d \epsilon / d \delta R$. Expanding $e^{-\epsilon^{\prime} \delta R / k_{B} T} \approx 1-\epsilon^{\prime} \delta R / k_{B} T$ yields $\delta \Psi \approx\left\{\left(N x_{0} \epsilon^{\prime} \delta R\right) /\right.$ $\left.\left[k_{B} T\left(1+x_{0}\right)^{2}\right]\right\}$, where $e^{-\mu / k_{B} T}=\zeta$ and $\epsilon\left(R_{0}\right)=\epsilon_{0}, x_{0}=$ $\zeta e^{-\epsilon_{0} / k_{B} T}$. Thus, changes in $R$ also drive changes in $\Psi$, which evolves to its final value, given the change $\delta R$, through a kinetic coefficient that multiplies the term above. Adding the term in $\delta \Psi$ coming from the epigenetic potential, which can be assumed to be quadratic at lowest order in an expansion about the stable value $\Psi_{0}: V(\delta \Psi)=$ $\frac{1}{2} A(\delta \Psi)^{2}$, we have our final result: $\dot{\Psi}=-A \delta \Psi+B \delta R$. Here, $A$ is simply the inverse of the relaxation time for compaction fluctuations $\tau_{\Psi}$, defined below. The sign of $B$ depends on the sign of $\epsilon^{\prime}$ since all other quantities that enter its definition $\left(N, x_{0}\right.$, and $\left.T\right)$ are explicitly positive, reducing to the question of whether the nucleosome binding energy is reduced when the nucleus is expanded. In general, as is known from in vitro single-molecule experiments, extending DNA expels bound nucleosomes, implying that their binding energy is reduced upon stretching; thus, the sign of $B$ should be negative for the auxetic state, given our interpretation above. More details of the physical arguments are provided in Ref. [49].

\section{Estimation of parameters}

We now estimate $C=4 \kappa K_{a} / \mu L R_{0}^{2}$ and $D=\kappa k_{B} T^{*} \phi_{0}^{2} /$ $\mu L \nu_{c} \chi^{\prime}$. We take $\kappa=l_{p}^{2}$, relating the permeability $\kappa$ to the pore size $l_{p}$. Assuming a nuclear pore complex size of $l_{p} \simeq$ $5 \mathrm{~nm}$ [50], this yields $\kappa=2.5 \times 10^{-17} \mathrm{~m}^{2}$. From plate theory, the area modulus $K_{a}$ and the Young's modulus $E$ are related through $K_{a} \approx E t$ [30], where $t$ is the thickness of the plate. Thus, $C=\left[\left(4 \kappa \kappa_{A}\right) /\left(\mu L R_{0}^{2}\right)\right] \approx\left[\left(4 l_{p}^{2} \times E t\right) /\right.$ $\left.\left(\mu t R_{0}^{2}\right)\right]=\left[\left(4 l_{p}^{2} E\right) /\left(\mu R_{0}^{2}\right)\right]$. With $E \approx 200 \mathrm{~Pa}$ [51-53], the radius of the nucleus $R_{0}=5 \times 10^{-6} \mathrm{~m}$ and $\mu \approx 2-3$ centipoise $\approx 2 \times 10^{-3} \mathrm{~Pa}$ - sec [54], $C \approx 0.4 \mathrm{sec}^{-1}$. To calculate $D$, we assume that the length over which the pressure drops is the same as the membrane thickness (65 $\mathrm{nm}$ [55]). With $\nu_{c}=(10 \mathrm{~nm})^{3}$ [56-58], a polymer volume fraction of approximately 0.1 [59] (but see also Refs. [60,61]), and $T^{*} \simeq 300 \mathrm{~K}$, we obtain $D \approx 8 \times 10^{-6} \chi^{\prime} \mathrm{m} / \mathrm{sec}$. Our estimate for the polymer volume fraction is discussed further in the Appendix A.

\section{E. Mapping to the experimental system}

In a steady state, $\delta \Psi=[(-B f) /(2 A C+3 B D)], \delta R_{\|}=$ $-[(2 A C+2 B D) /(2 A C+3 B D)](f / C)$, and $\delta R_{\perp}=[(B D) /$ $(2 A C+3 B D)](f / C)$. For finiteness, we require $2 A C+$ $3 B D \neq 0$. From this requirement, the Poisson's ratio is $\nu=-\left[\left(\delta R_{\perp}\right) /\left(\delta R_{\|}\right)\right]=[(B D) /(2 C A+2 B D)]$. Choosing the experimental value of $\nu=-0.25$ and rearranging the above expression, we find that $[(C A) /(B D)]=-3$. Making a reasonable choice for the ratio $\tau_{\Psi} / \tau_{R} \simeq 0.01$ yields $\tau_{\Psi}$, and the value of $C$ obtained above yields $\tau_{R}=$ $2.5 \mathrm{sec}$ and $\tau_{\Psi}=2.5 \times 10^{-2} \mathrm{sec}$, with $A=40 \mathrm{sec}^{-1}$. We obtain $B=-[(C A) /(3 D)]=-\left[\left(7 \times 10^{5} \times 5 \times 10^{7}\right) /\left(3 \chi^{\prime}\right)\right]=$ $-\left[\left(7 \times 10^{5}\right) /\left(\chi^{\prime}\right)\right] \mathrm{m}^{-1} \mathrm{sec}^{-1}$. Our final set of parameter values is then $C=0.4 \mathrm{sec}^{-1}, \quad \tau_{R}=2.5 \mathrm{sec}, \quad D=$ $8 \times 10^{-6} \chi^{\prime} \mathrm{m}-\mathrm{sec}^{-1}, A=40 \mathrm{sec}^{-1}, \tau_{\Psi}=2.5 \times 10^{-2} \mathrm{sec}$, $B=-\left[\left(7 \times 10^{5}\right) / \chi^{\prime}\right] \mathrm{m}^{-1} \mathrm{sec}^{-1}$. In dimensionless units, taking $\tau_{\Psi}=2.5 \times 10^{-2} \mathrm{sec}$ and measuring the length in units of $R_{0}$ yields $A=1.0, C=0.01, D=0.04 \chi^{\prime}$, and $B=-0.09 / \chi^{\prime}$. If we assume $\left.(d \chi / d \delta \Psi)\right|_{\Psi=\Psi_{0}} \simeq\left(\chi / \Psi_{0}\right)$, $\chi \approx 0.5$, and $\Psi_{0}=1$, we obtain $\chi^{\prime}=0.5$.

\section{F. Numerical simulations}

Our numerical simulations implement Langevin dynamics in solving the stochastic equations (1). We use both a simple Euler discretization and a fourth-order Runge-Kutta method, checking that both give essentially similar results.

\section{RESULTS}

\section{A. Auxetic and normal mechanical behavior in a model description of nuclear indentation}

The AFM indentation experiment corresponds to taking $f=f_{\|} \neq 0$ and setting $f_{\perp}=0$. The set of model equations (1) have a number of parameters, which we fix using experimental and theoretical input. The choice of parameters and the range of values they can take are discussed in Sec. II. The solutions of these equations are provided in Appendix B.

Figure 2 shows plots of $\delta \Psi, \delta R_{\|}$, and $\delta R_{\perp}$ [Fig. 2, panels (e)-(h)] for small $f$, as obtained from our model equations. The quantities $\delta \Psi, \delta R_{\|}$, and $\delta R_{\perp}$ vary linearly with $f$, a consequence of the fact that we assume that $V(\delta \Psi)$ increases quadratically about its stable value. This is across the parameter values shown in Figs. 2(a)-2(d), for the state points $(B, C)$ marked on the figures with the filled black circle. These plots are for a choice of parameters corresponding to nonauxetic, i.e., regular behavior. For the normal, i.e., nonauxetic state, the slopes of $\delta R_{\|}$and $\delta R_{\perp}$ vs $f$ should have opposite signs.

Our model predicts that the slope of $\delta \Psi$ vs $f$ is negative; i.e., the compaction decreases with the applied force in the nonauxetic state; see Fig. 2, panels (e) and (h).

In Figs. 2(m)-2(p), we also show results for the auxetic case, where the slopes of $\delta R_{\|}$and $\delta R_{\perp}$ vs $f$ have the same 


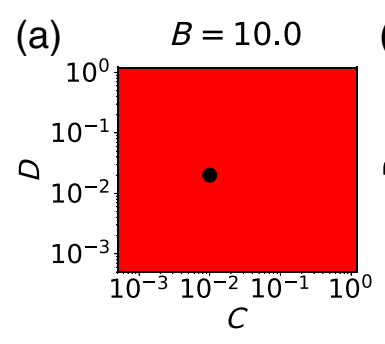

(e)
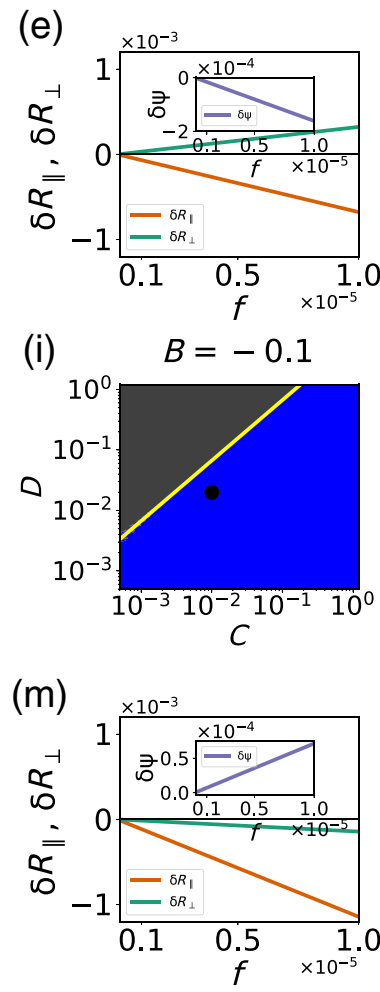

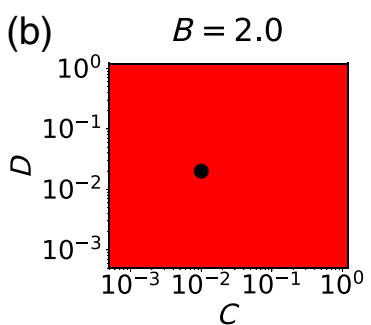

(f)
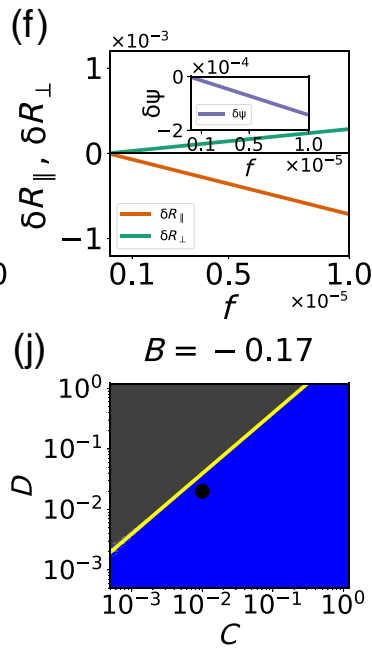

(n)

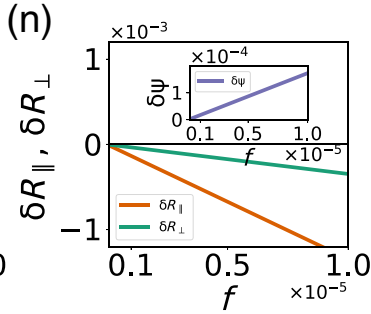

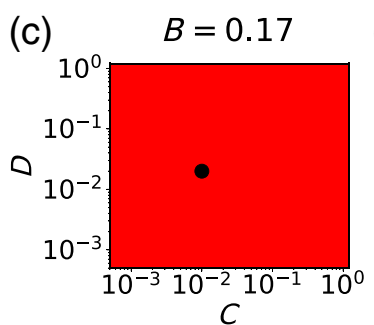

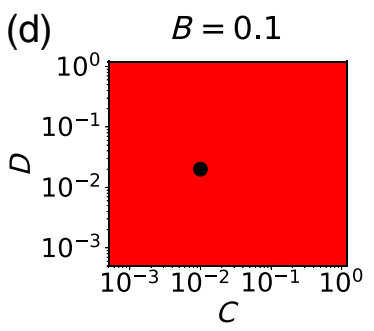

(g)
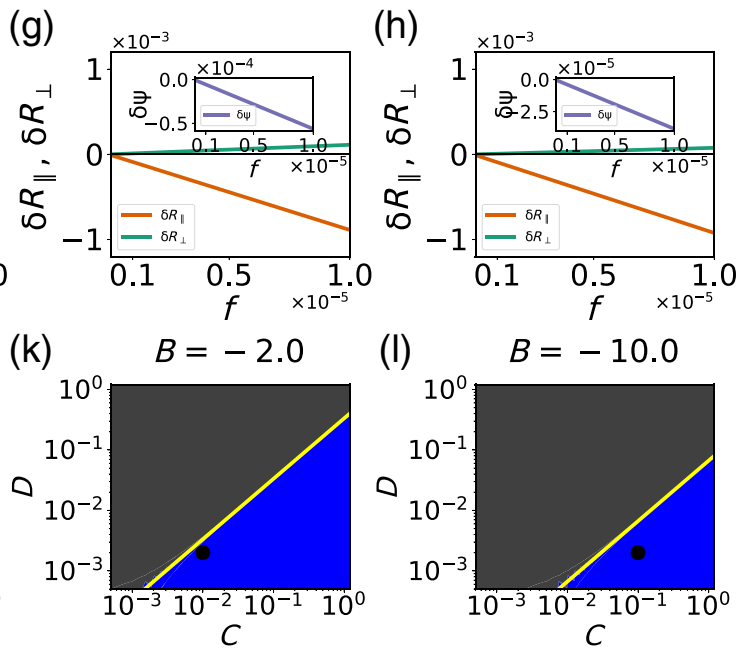

(o)

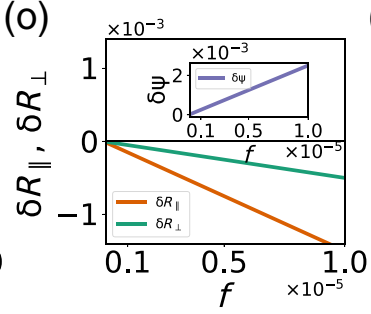

FIG. 2. Parameter choices for $C$ and $D$ : (a) $B=10.0$, (b) $B=2.0$, (c) $B=0.17$, (d) $B=0.1$, all in the regime of nonauxetic (regular) behavior. We show the behavior of the dynamical variable with the increase in force for the parameter values (e) $C=$ $0.01, D=0.02, B=10.0$; (f) $C=0.01, D=0.02, B=2.0$; (g) $C=0.01, D=0.02, B=0.17$; (h) $C=0.01, D=0.02, B=0.1$. Parameter choices for $C$ and $D$ are shown in the auxetic regime with (i) $B=-0.1$, (j) $B=-0.17$, (k) $B=-2.0$, (1) $B=-10.0$. The line separating the blue and gray regions marks the stable-unstable boundary. We show the behavior of the dynamical variable with the increase in force for the parameter values (m) $C=0.01, D=0.02, B=-0.1 ;$ (n) $C=0.01, D=0.02, B=-0.17 ; \quad($ o) $C=0.01, D=0.002, B=-2.0$; (p) $C=0.1, D=0.002, B=-10.0$. [Red and blue show the regions where a stable solution is obtained $($ red $=$ normal, blue $=$ auxetic), while grey shows where solutions become unstable].

sign. Note that $\delta R_{\|}$and $\delta R_{\perp}$ now both decrease with $f$. This result indicates auxetic behavior. This behavior is seen across the parameter values shown in Figs. 2(i)-2(1), for the state points marked on the figures with the filled black circle. These parameter values are chosen in the regime where the fixed point is stable, shown in blue. (The grey region shows the regime in which the equations have unstable solutions.) In the auxetic case, the slope of $\delta \Psi$ vs $f$ is positive.

Thus, the solutions of our model equations yield both auxetic and nonauxetic behavior, controlled by the sign of $B$ in Eqs. (1). The results are consistent with the schematics of Figs. 1(f) and 1(g), which show how chromatin compaction varies upon the application of an external force.
The additional information they provide relates to the behavior of the compaction variable. As shown in Sec. II, the parameter values we derive are consistent with experimental measures of auxeticity in transitional stem-cell nuclei.

\section{B. Describing nuclear shape changes in microchannel flow}

Nuclear indentation through the AFM method described in Ref. [34] provides a direct way of accessing the auxetic mechanical behavior of the nucleus. Here, a fixed force is applied along the longitudinal $(\|)$ direction, and a transverse $(\perp)$ deformation is measured. An alternative method 
involves an optofluidic assay, in which cells are passed through narrow microchannels of controlled width. These cells are then imaged through fluorescence microscopy of Syto13 labeled cells. When the width of the channel is comparable to the cell size, cell dimensions are constrained. A further complication is the role of stretching stresses caused by cytoskeletal strain acting when cells are confined to the microchannel. Given our model assumptions, we may model the confined case by accounting for both $f_{\|}$and $f_{\perp}$ in the governing equations, Eqs. (1). Whereas $f_{\|}$is primarily controlled by the size of the constriction through which these cells pass, $f_{\perp}$ derives from the anisotropic remodeling of the actin cytoskeleton.

The geometry of the microchannel experiments is shown in Fig. 3(a), where we show a cell confined to a channel whose width is comparable to cell dimensions. In the experiments, the channel width is $12 \mu \mathrm{m}$, while cell sizes range from $6 \mu \mathrm{m}$ to $14 \mu \mathrm{m}$. Figure 3(b) shows a schematic of the effects of the combination of longitudinal and transverse forces applied to cells of different sizes, as obtained in our calculations; see below. For small cells in the auxetic case, if they have unconfined dimensions much smaller than the channel width, their longitudinal and transverse dimensions increase when they are confined to the channel. For larger cells in the same limit, both dimensions decrease. These results are consistent with expectations from auxetic behavior. On the other hand, irrespective of cell sizes in the nonauxetic case, the longitudinal dimension decreases while the transverse dimension increases. These results are consistent with the behavior shown in Fig. 3(b). These schematic results recapitulate the results of Ref. [34].

To extend this case to the mechanical response of stem cells of various sizes in a microchannel, our modeling strategy is the following. The experiments, performed on a range of cell sizes at a fixed microchannel width, obtain longitudinal and transverse strains for an ensemble of cells of different sizes. At the extreme limits of cell sizes, Fig. S10 of Ref. [34] shows averaged strains in the parallel and perpendicular directions. These strains are proportional to $R_{\|}$and $R_{\perp}$ in our definitions in Eq. (1), and using $R_{0}$ as our unit of length converts this proportionality to an equality. We label these strains as $S_{\|}$and $S_{\perp}$ and display them in Fig. 3(c), (I)-(III), for the transitional, primed, and naive ES cell states. Starting with these results, we can invert the relationship between strains and forces, finding the effective $f_{\|}$and $f_{\perp}$ that produce these strains.

We can now explore the space of values of $\left(f_{\|}, f_{\perp}\right)$, constructing contour plots of $\delta \Psi, \delta R_{\perp}$, and $\delta R_{\|}$, as shown in Fig. 3(d), (I)-(III). The parameters chosen are for the smallest and the largest cells, using the data shown in Fig. 3(c), (I). The solid lines in Fig. 3(d), (II) and (III), represent a choice of a few lines of constant strain in each case, as a function of $f_{\|}$and $f_{\perp}$. These lines then predict the forces $\left(f_{\|}, f_{\perp}\right)$ required to create a fixed strain across cells of different sizes.

The extremal points of Fig. 3(b), (I)-(III), are now associated to points on the $\left(f_{\|}, f_{\perp}\right)$ surface. We can now model the data for cells of intermediate sizes by supposing that $f_{\|}$and $f_{\perp}$ vary independently and linearly between their terminal values. We ask if these results can fit data for intermediate cell sizes, shown in the scatter plot illustrated in Fig. 3 of Ref. [34]. These results are shown in Fig. 3(e), (I)-(III), for transitional, primed, and naive ES cell states. The experimental data are shown as points, while the theoretical prediction that follows from our analysis is shown as the green line. In all three cases, there is an approximate linear relationship between $S_{\|}$ and $S_{\perp}$ that our calculation captures. The magnitude of the strains at intermediate values of cell size is correctly rendered.

Thus, our model, despite its simplicity, captures all essential features of the data of Ref. [34]. As we have pointed out, the model can then be used to provide specific predictions for mechanical response in cells of different sizes. Also, even though the chromatin compaction variable $\delta \Psi$ was not measured in those experiments, our model provides specific predictions for how this quantity varies across different cell sizes in comparison to the width of the microchannel. This prediction is experimentally testable, and we discuss possible ways of doing so in the concluding section.

\section{Autocorrelations and cross-correlations of chromatin compaction and nuclear dimensions in the auxetic regime}

The previous sections explored the use of an external force, either applied directly using an AFM tip or indirectly by confining cells to a narrow microchannel, in understanding auxetic and nonauxetic behavior. However, our general model formulation suggests how less-invasive ways of probing the coupled mechanical response of chromatin compaction and nuclear dimensions might provide useful information. Let us assume that we can measure both chromatin compaction and the dimensions of the nucleus simultaneously as a function of time-possible ways of doing this are discussed later. Assuming an initially spherical nucleus, $R_{\|}$and $R_{\perp}$ coincide since now there is no externally imposed direction that leads to an anisotropic mechanical response. The only relevant mechanical variable is then $R(t)$, the radius of the spherical nucleus as a function of time. Our equations are now simpler since they involve only the two variables $\Psi$ and $R$. The solution to the full set of equations is provided in Supplementary Information.

Given measurements of $\Psi(t)$ and $R(t)=R_{0}+\delta R(t)$, we can ask whether signatures of auxetic and nonauxetic behavior might be visible in such measurements. Since such measurements provide data in time, we can compute 
(a)

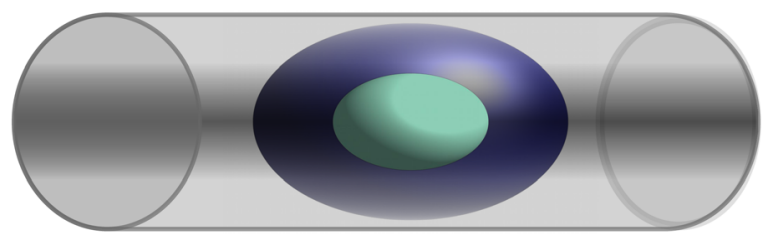

(c)

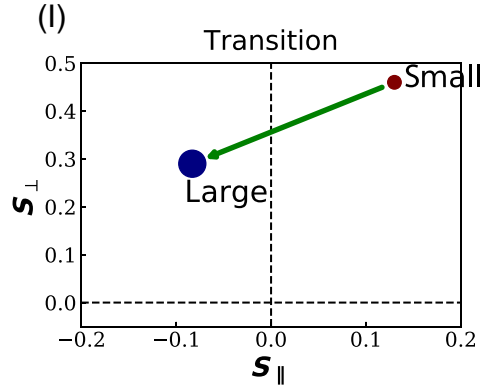

(d) (l)

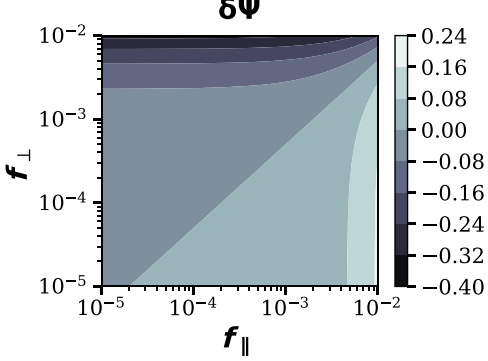

(e)

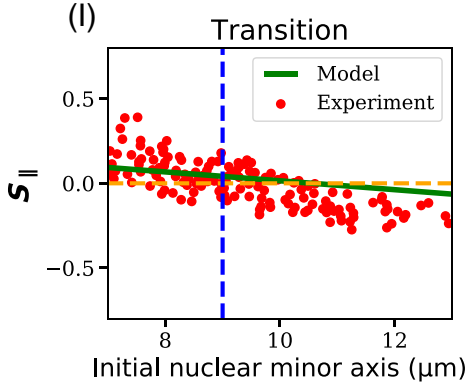

(II)

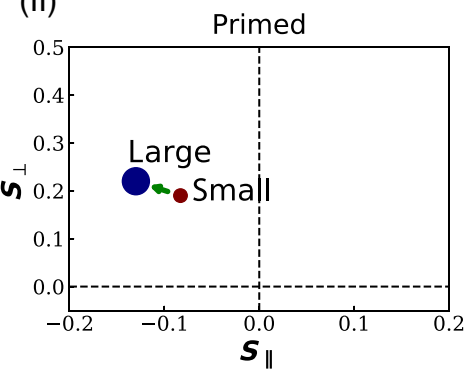

(II)
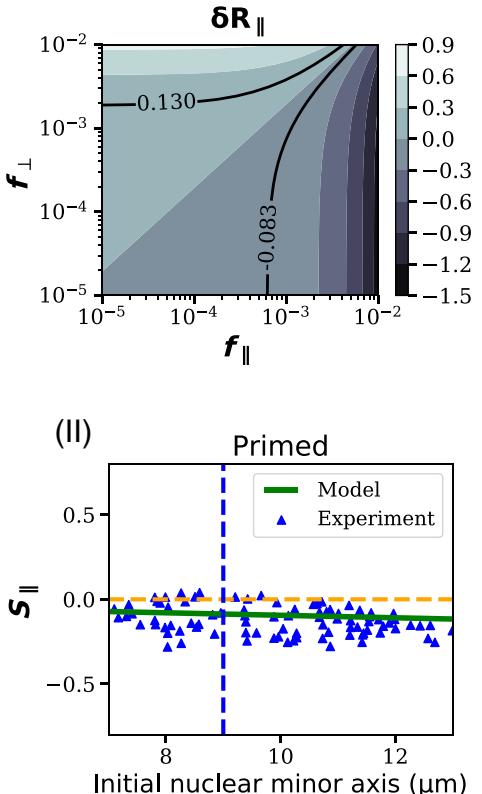

Auxetic Nonauxetic

(b)

Small

nuclei

Large nuclei

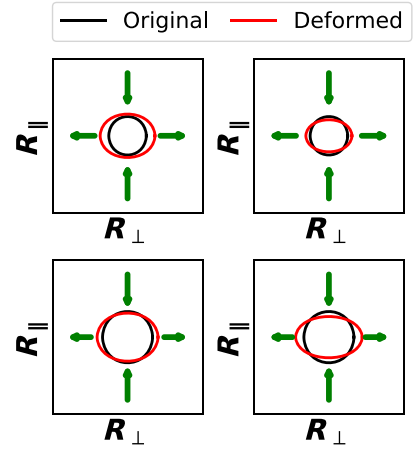

(III)

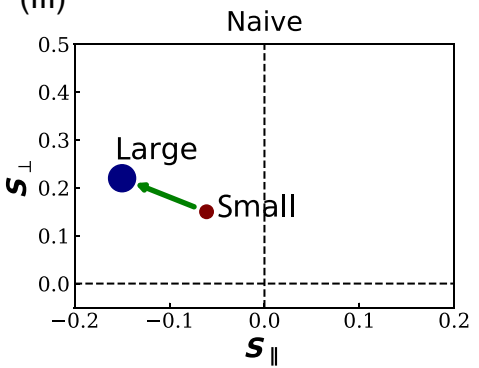

(III)
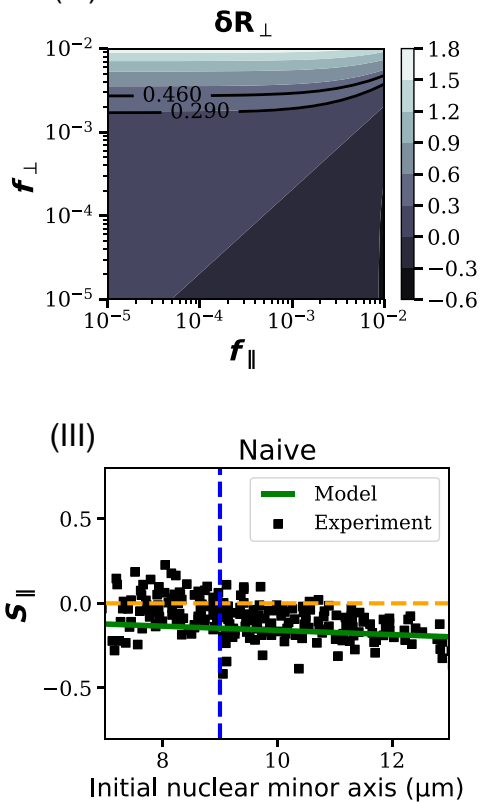

FIG. 3. (a) Schematic of a cell confined to a microchannel with width comparable to cell dimensions. (b) Schematic of the effects of the combination of longitudinal and transverse forces applied to cells of different sizes. These effects follow from our calculations and are consistent with the results of Ref. [34]. (c) Plots of $S_{\|}$and $S_{\perp}$ extracted from experiments, for the transitional, primed, and naive ES cell states. The arrow connects the two terminal points. (d) Contour plots for $\delta \Psi, \delta R_{\|}$, and $\delta R_{\perp}$, against $\left(f_{\|}, f_{\perp}\right)$, with solid lines showing loci of constant strain. (e) Predictions for transitional, primed, and naive ES cell states, of $S_{\|}$and $S_{\perp}$. The straight line represents experimental predictions for intermediate cell sizes. Experimental data are digitized from the scatter plot of Fig. S10 of Ref. [34] and shown on the same figure.

autocorrelations of these variables as well as their crosscorrelations. The solutions to these equations can be computed explicitly and are provided in Supplementary Material. Figure 4 shows our computation (lines show exact calculations; points are numerical solutions of the Langevin equation) of autocorrelations and crosscorrelations in the model, with parameters chosen within the auxetic regime. 

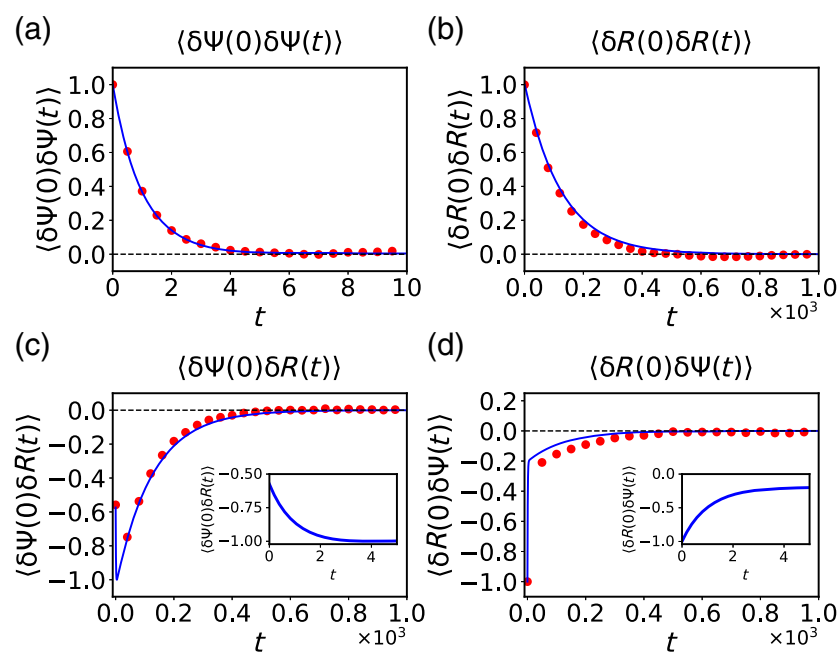

(d)

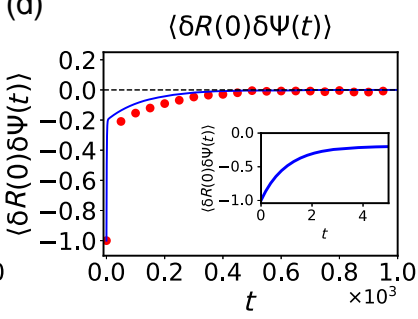

FIG. 4. Computations of autocorrelations and cross-correlations in the simplified two-component model, in the auxetic regime, with $C=0.01, D=0.02$, and $B=-0.17$. We illustrate the calculation of the following correlation functions: (a) the autocorrelation of the $\delta \Psi$ variable, $\langle\delta \Psi(0) \delta \Psi(t)\rangle$, (b) the autocorrelation of the $\delta R$ variable, $\langle\delta R(0) \delta R(t)\rangle$, (c) The crosscorrelation between $\delta \Psi$ and $\delta R,\langle\delta \Psi(0) \delta R(t)\rangle$, and (d) the crosscorrelation of $\delta R$ and $\delta \Psi,\langle\delta R(0) \delta \Psi(t)\rangle$. The insets show the behavior close to the origin in two special cases where there is a competition between the two timescales for relaxation. Points represent the numerical solution of the Langevin equations, while lines represent the analytic formulas.

The autocorrelations $\langle\delta \Psi(0) \delta \Psi(t)\rangle$ and $\langle\delta R(0) \delta R(t)\rangle$ are shown in Figs. 4(a) and 4(b), whereas the crosscorrelations $\langle\delta \Psi(0) \delta R(t)\rangle$ and $\langle\delta \Psi(0) \delta R(t)\rangle$ are shown in Figs. 4(c) and 4(d), respectively. The insets expand the behavior of the cross-correlation functions close to the origin, where two timescales for relaxation compete. The timescale for the relaxation of autocorrelations in the $\Psi$ variable is substantially smaller than for the $R$ variable. The cross-correlations $\langle\delta \Psi(0) \delta R(t)\rangle$ and $\langle\delta \Psi(0) \delta R(t)\rangle$ both relax to zero in an interesting two-step process, with a sharp initial step reflecting the relaxation of the fast variable $\Psi$ followed by a slower relaxation, primarily driven by the $R$ variable.

We can further investigate (see Supplementary Information) model predictions for the case in which a weak force is applied and allowed to vary in time in a sinusoidal fashion. For our linear system of equations, this then implies that quantities such as $\Psi, R_{\|}$, and $R_{\perp}$ should also oscillate at the same frequency, but with a phase lag between them. This phase lag predicts the relative importance of what is termed reactive and dissipative response, with the first largely located in the elastic properties of the nuclear envelope and the second associated to dissipation connected to the flow of fluid across the nuclear envelope as well as of the friction encountered by chromatin as its fluctuations relax. These can be predicted from the theoretical formulation and indeed are the focus of standard experiments in the physics literature that studies soft materials, but whether their experimental analogue can be probed in biophysical measurements on stem cells is an open question.

Extracting behavior as shown in Fig. 4 would constitute a powerful test of model predictions.

\section{Correlations across the auxetic-nonauxetic boundary as probes of the transition}

Our model describes chromatin compaction states using a single variable $\Psi$, with larger values of $\Psi$ representing overall more compact states of chromatin packing. We propose that $\Psi$ fluctuates in time about an approximately constant value but that these fluctuations are constrained by a chromatin compaction potential defined as $V(\delta \Psi)$, which controls how large they can be. These fluctuations are also constrained by their coupling to nuclear dimensions through the variables $\delta R_{\|}$and $\delta R_{\perp}$. They are influenced, as well, by the inherent noisiness of nucleosome on-off dynamics in a hyperdynamic state. All these effects are included in our model.

This choice of a "chromatin compaction potential" identifies the relevant biophysical distinction between more open, gene-rich euchromatin and more tightly bound, genepoor heterochromatin as broadly being one of local compaction. We project the multidimensional landscape of potential chromatin states that Waddington envisaged, which should be more generally describable through a spatially varying and sequence-dependent compaction field, onto a single scalar compaction variable. Our equations then provide a way of understanding how such a compaction variable couples to mechanical variables describing nuclear shape and size.

Our results suggest a simple method for determining the location of the auxetic-to-nonauxetic transition. We work in the limit described in the previous section, where we infer the transition by monitoring the system noninvasively, measuring only the variables $\Psi$ and $R$ as functions of time in a steady state. From these measurements, we can calculate their autocorrelations and cross-correlations.

In Fig. 5, we show plots of the correlation functions $\langle\delta R(0) \delta \Psi(t)\rangle$ and $\langle\delta \Psi(0) \delta R(t)\rangle$. These plots illustrate that $\langle\delta R(0) \delta \Psi(t)\rangle$ is a good indicator of the transition from auxetic-to-nonauxetic behavior, with $\langle\delta R(0) \delta \Psi(t)\rangle$ changing the sign of its slope upon approaching its asymptotic value across the auxetic-to-nonauxetic boundary. On the boundary, there is no correlation at all, to this order, between fluctuations in the nuclear dimension and fluctuations in chromatin compaction. Since the change from auxetic-to-nonauxetic behavior is marked by the parameter $B$ changing sign, it must cross zero at least at one point. (Since the experimental sequence encountered as ES cells differentiate is nonauxetic $\rightarrow$ auxetic $\rightarrow$ nonauxetic, this suggests that $B$ should change sign at least twice. 
(a)

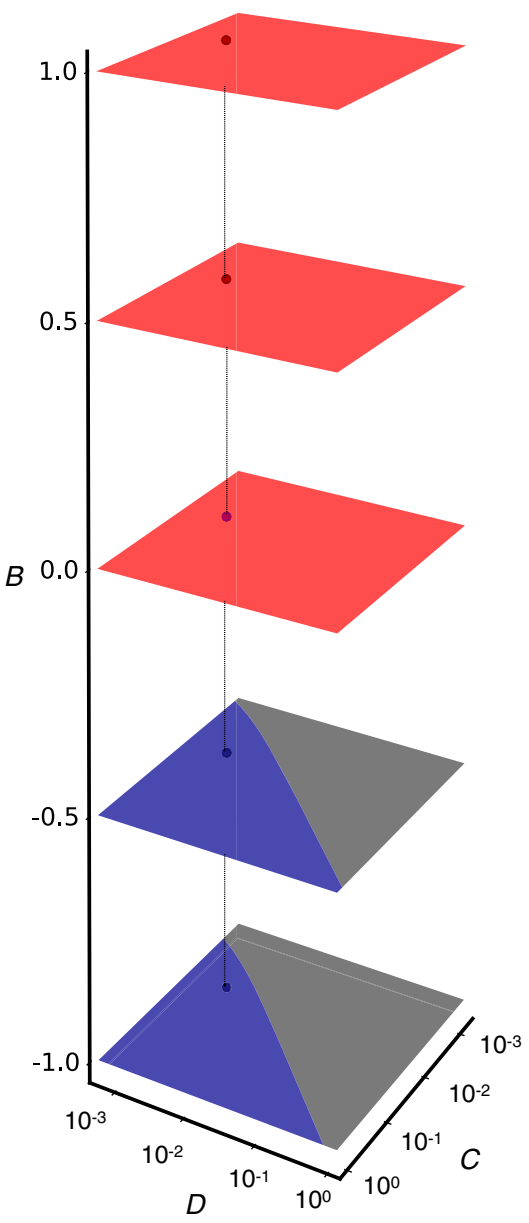

(b)
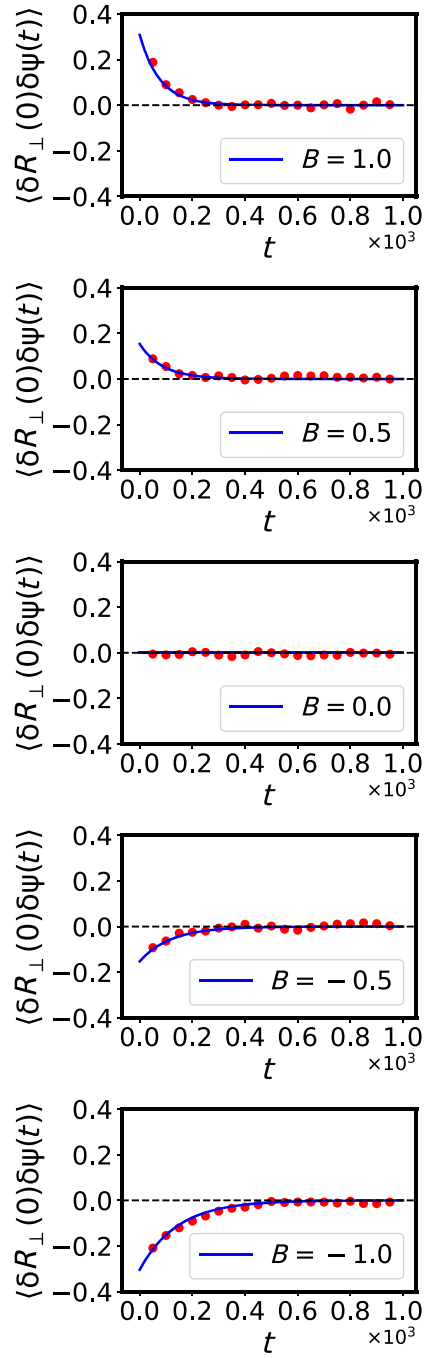

(c)
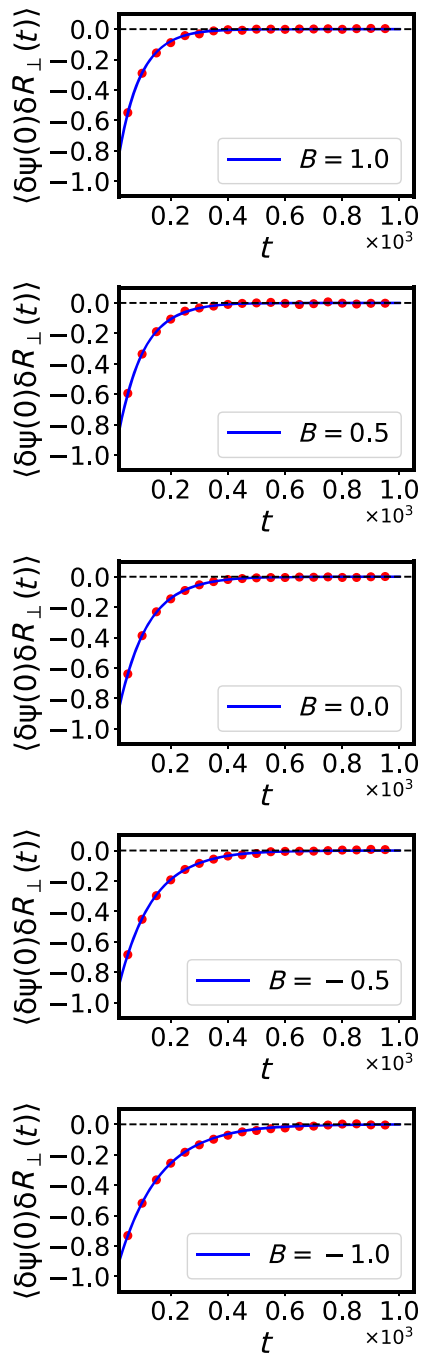

FIG. 5. (a) Our choice of parameters in $(B, C, D)$ space, with $B$, shown on the vertical axis, varied so as to cross the auxetic-tononauxetic boundary. Columns (b) and (c) illustrate the correlation functions $\langle\delta R(0) \delta \Psi(t)\rangle$ and $\langle\delta \Psi(0) \delta R(t)\rangle$. Across the auxetic-tononauxetic boundary, where the sign of $B$ changes, the $\Psi$ variable decouples, at lowest order, from the $\delta R$ variable, leading to a flat behavior of the correlation $\langle\delta R(0) \delta \Psi(t)\rangle$. In contrast, while $\Psi$ is not influenced by $\delta R$, fluctuations in $\Psi$ do couple to $\delta R$, leading to a nontrivial relaxation of the correlation function $\langle\delta \Psi(0) \delta R(t)\rangle$. This change of sign of $d\langle\delta R(0) \delta \Psi(t)\rangle / d t$ indicates that the auxetic to nonauxetic boundary has been crossed.

This specific prediction can be addressed in experiments, as we discuss below.)

Now, note that at this special point, fluctuations in $\Psi$ decouple from fluctuations in the nuclear-size variable to linear order; fluctuations in $\Psi$ influence fluctuations in $\delta R$ but not vice versa. As we show below, this case provides a practical way of accessing $V(\delta \Psi)$.

\section{E. Inferring $\boldsymbol{V}(\delta \Psi)$ from experimental data}

We can describe the transition between ESC, T-ESC, and differentiation-primed states in terms of a trajectory in the space of the variables $C, D$, and $B$. As is standard, we can assume that the parameters controlling these variables must vary smoothly since they reflect continuous shifts in the transcriptome; indeed, the assumption of smooth variation is central to landscape ideas. As the stem cell transits between these states, it encounters auxetic $(B<0)$ [Figs. 6(g)-6(i)] and nonauxetic $(B>0)$ [Figs. 6(d)-6(f)] states, with an intervening $B=0$ state [Figs. 6(a)-6(c)].

For each of these chosen values of $B$, we illustrate the choice of a specific chromatin compaction potential that we can model as a smooth function, shown via the solid lines in each subplot. We choose these functions to be (1) a simple quadratic potential, (2) a quartic potential with a shallow minimum at the origin and two symmetrically placed deeper minima on either side, and (3) the more complex case of a quadratic potential with a superposed sinusoidal 
- Assumed potential $\begin{aligned} & \text { Reconstructed potential } B=0.0 \\ & \text { Reconstructed potential } B=1.0 \\ & \text { Rected potential } B=-1.0\end{aligned}$

(a) $\quad V(\delta \psi)=\frac{a}{2} \delta \psi^{2}$

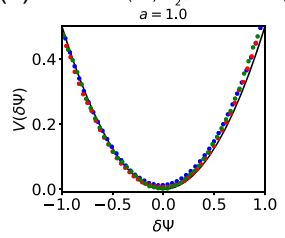

(b) $\quad V(\delta \psi)=\frac{a}{2} \delta \psi^{2}+\frac{b}{4} \delta \psi^{4}+\frac{c}{6} \delta \psi^{6}$

(c)
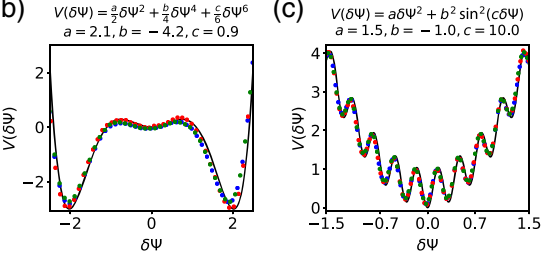

FIG. 6. Reconstruction of the potential landscape $V(\delta \Psi)$ in the simplified two-component model: (a) assuming a quadratic potential, (b) assuming a sixth-order potential, and (c) assuming a quadratic potential with a superimposed sinusoid- $V(\delta \Psi)=$ $a \delta \Psi^{2}+b^{2} \operatorname{Sin}^{2}(c \delta \Psi)$, for $B=0.0$ (blue), $B=1.0$ (red), and $B=-1.0$ (green).

modulation that provides more intricate structure. We do not yet know what form such a potential takes in the experiments, but we intend to illustrate a method by which information from the measurement of fluctuations could help in its extraction.

For $B=0$, as shown in Figs. 6(a)-6(c) (blue filled circles), given that $\delta \Psi(t)$ reflects its relaxation in the chromatin compaction potential, we form a histogram of $\delta \Psi$ values. Since the governing equation for the $\delta \Psi$ variable can be interpreted as a Langevin equation for a particle moving in the specified potential, the steady-state probability distribution of $\delta \Psi$ can be inferred from this histogram in a straightforward manner, as discussed in Appendix C. Figures 6(a)-6(c) show results from a numerical and analytic reconstruction of the assumed potential $V(\delta \Psi)$ using such a method. In this way, we thus proceed from the histogram of measured values to the potential that controls such fluctuations. While the data used in these figures are "synthetic," the procedure for extracting the potential from them is robust.

Provided $|B| \neq 0$ is not too large and for parameter values comparable to the ones we use, this procedure reconstructs $V(\delta \Psi)$ reasonably well, a consequence of the fact that fluctuations in $\delta R$ couple relatively weakly to fluctuations in $\delta \Psi$. Figures 6(a)-6(c) show $V(\delta \Psi)$, obtained from histograms of $\delta \Psi$ values for $B=-1$ (red filled circles) and $B=1$ (green filled circles). These diagrams suggest that one need not precisely locate the region where $B$ vanishes for this approach to be of use.

\section{DISCUSSION}

An "epigenetic landscape," whose lowest points represent gene-expression patterns encoding specific differentiated states, is often pictorially represented in the following way [24,62]: Imagine projecting all possible gene-expression states onto a two-dimensional $(X Y)$ plane. This projection is constrained by the requirement that two nearby state points represent closely related expression patterns. (Naively, the rewiring of gene-regulatory networks required to convert expression programs from one cell type to another should be smaller the more similar these cell types are [24].) The height of a surface (the landscape) above a point on this plane can then be assigned to the relative "energy" of the state described by that point. The shape of the surface can then be used as a qualitative way of describing barriers to accessing different gene-expression patterns starting from a given initial state.

The plasticity required of gene-regulatory networks in the stem-cell states implies, in this pictorial analogy, that the shape of the landscape should determine which states will become unstable-and to which other states-as biochemical and mechanical parameters are changed. Biochemical parameters here could refer to levels of protein factors that modulate stemness, while mechanical parameters could represent the stiffness and anisotropy of the substrate on which these cells are cultured [63]. If one imagines, as Waddington did, a ball rolling on this landscape as representing the stem-cell state choosing between terminally differentiated states, the motion of the ball should be biased by the underlying shape of the landscape, including its peaks, ridges, and valleys. The resulting energy surface can be depicted as a geographical landscape, along the lines of Waddington's original picture.

Such a qualitative picture also suggests that this landscape might also be thought of as dynamic, tilting and deforming to favor one set of states over others. This would then describe how an initial state might be guided to a specific cell fate as the cell integrates external environmental signals when driven to differentiate.

The description of the previous paragraph proceeds along conventional lines. Our view here emphasizes biophysical aspects of this argument. Instead of projecting states depending on their proximity in gene-expression space, we imagine them to be projected according to their level of chromatin compaction; arguments concerning the proximity of closely related cell types in such a "chromatin compaction" space should parallel those in the case of the "gene-expression" space. To motivate this case, we note that the relative ratio of heterochromatin to euchromatin varies across differentiated cell types [64]. It has been suggested that chromatin density might itself act to regulate gene expression in a stem-cell population [65]. While the embryonic stem-cell state has a chromatin organization that is best described as a highly correlated fluid, the differentiated state fluctuates far less, with condensed heterochromatin foci forming during the differentiation of pluripotent embryonic stem cells [66]. The formation of heterochromatin domains has recently been argued to be mediated by phase separation $[39,40]$. Together with the accumulation of silencing histone marks, this results in differential expression [67]. Classifying the epigenetic states underlying these cell types through their levels of local chromatin compaction should provide one approximate way of connecting the theoretical ideas presented here to experimental data. 


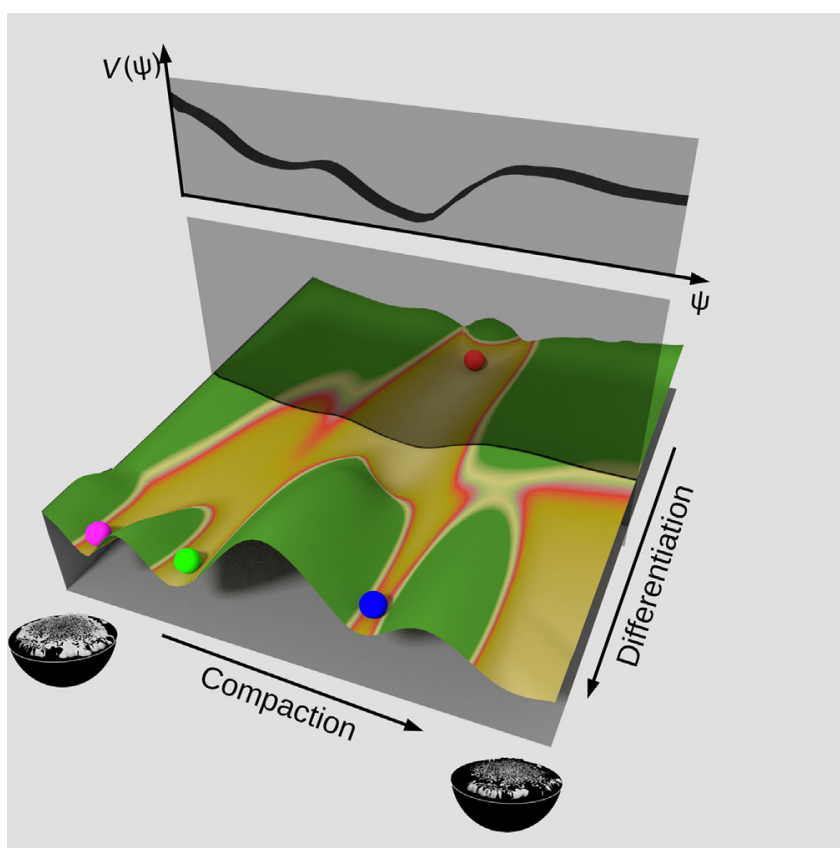

FIG. 7. Schematic of an epigenetic landscape in the compaction variable. A pictorial representation of the epigenetic landscape, projected onto a single variable describing overall compaction. Points towards the back of the figure represent the ES cell state, while points in the valleys towards the foreground represent differentiated states. As one moves from back to front, the figure describes how the effective potential governing overall compaction can be described via a cut through the landscape as shown.

This idea is illustrated in Fig. 7, which shows a schematic of such a landscape. The colored balls towards the front of the figure represent stable, differentiated states. The ball at the back represents the ES cell state. As the cell differentiates, one imagines that the landscape is tilted forward so as to allow the ball to fall towards these stable states. All possible accessible intermediate states can be represented, again pictorially, in terms of a plan that intersects this landscape. The curve that defines where these two curves intersect provides a one-dimensional surface, to be identified with the $V(\delta \Psi)$ of our discussion.

We stress that all projections from a high-dimensional space to a low-dimensional one involve a loss of information. The question is whether the reduced information that results from projecting the complexity of epigenetic control into the reduced space of overall compaction suffices for a biophysical description. Expanding the potential $V(\delta \Psi)$ about a local minimum led to the results described in this paper. However, we should ideally think of this potential itself as evolving over some timescale and the choice of the initial point as reflecting a cell-specific initial condition, such as cellular levels of Lamin A [68].

\section{CONCLUSIONS}

In this paper, we presented a theory of auxetic behavior in the nuclei of stem cells in the transitional state. We began by pointing out that the unusual mechanical properties of the stem-cell nucleus, as well as its fluctuations, should provide a window into the packaging and dynamic character of the chromatin states contained within it. We proposed that fluctuations in chromatin compaction should couple to fluctuations in the dimensions of the relatively soft nucleus that characterizes stem cells. We used these observations to argue that these coupled fluctuations, in chromatin packaging and nuclear shape, were most easily described in terms of a coupled, in general, nonlinear, dynamical system in three variables. We exploited the experimental observation that chromatin is least compact in the transitional state as compared to the pluripotent state and the differentiation primed state, to argue for a specific sign of the coupling term that connected size fluctuations to chromatin density fluctuations. We then showed how auxeticity resulted as a consequence, providing a simple and intuitive explanation for this puzzling observation. We then went on to suggest that we could map out the normalto-auxetic transition using ideas from the model. We further suggested experiments that could implement and test these ideas.

Our model could be generalized in several ways. The chromatin compaction variable is central to our discussion. Neglecting spatial variations in $\Psi$, as we do in this paper, should be a valid first approximation in the relatively fluid stem-cell state. However, chromatin compaction is certainly inhomogeneous in the differentiated state, while fluctuations about even the uniform state should, in principle, also be allowed for. Replacing $\Psi$ by the field variable $\Psi(\mathbf{r}, t)$, thus allowing it to vary both in space and in time, would be the logical next step in generalizing our model. Our simple parametrization of nuclear shape and mechanics could certainly be improved upon. Finally, simulations of a suitably coarse-grained model for active confined polymers, coupled to a flexible confining nuclear envelope, are feasible. It would be interesting to see how the arguments we supply here might be tested in such simulations. In all, however, we would not expect these technical improvements to alter our basic intuition qualitatively, and we would argue that the simplicity of our model makes it especially attractive.

We have proposed that projecting the complex spatialtemporal distribution of chromatin compaction onto an overall compaction variable and interpreting the time dependence of this variable in terms of motion within a simplified one-dimensional potential should provide a particularly useful biophysical way of formalizing Waddington's intuitive picture of an "epigenetic landscape" [22]. This way of understanding landscape ideas in the differentiation of stem cells is novel. Implementing the related analysis experimentally seems feasible. In particular, the fluorescence anisotropy measurements of labeled histones in the embryonic stem-cell state presented in Ref. [45], coupled to confocal microscopy measurements 
of the nuclear dimensions, should provide a noninvasive way of determining the coupling of chromatin compaction to mechanical variables describing the nucleus and its shape. Examining other possibilities for simultaneously characterizing chromatin compaction in addition to nuclear size and shape in a noninvasive way would be especially valuable.

Connecting microscopic, molecular-scale biochemical views of how stem-cell transcriptional programs are modulated, with the averaged, larger-scale biophysical approach that we describe in this paper, should lead to an improved understanding of the communication between stem-cell nuclear mechanics and chromatin states. This improved understanding would also help to illuminate the role of the mechanical environment in biasing lineage choice.

\section{ACKNOWLEDGMENTS}

K. T. thanks Ankit Agrawal and Rishu Kumar Singh for useful discussions. Both authors thank M. Muthukumar for valuable input. G. I. M. has benefited from conversations with J-F. Joanny, Aparna Baskaran, Sriram Ramaswamy, and Maxime Dehan. G. I. M. is also grateful for support from the Shastri Indo-Canadian Institute under the Shastri Mobility program.

This research was designed by G. I. M. Both authors (K. T. and G. I. M.) performed research and computations. K. T. plotted figures. Both authors contributed to writing the manuscript.

The authors declare no competing interests.

\section{APPENDIX A: VALUE OF CHROMATIN VOLUME FRACTION}

To calculate the chromatin volume fraction, we approximate DNA as a cylinder and the histone octamer as a disc and calculate the volume of all the components. The number of base pairs in the mouse genome is approximately $3 \times 10^{9}$. The average size of a base pair is approximately $3.4 \AA$ [69]. Thus, the length of DNA is $\left(3.4 \times 10^{-10}\right) \times\left(3 \times 10^{9}\right) \sim 1 \mathrm{~m}$ for the haploid case. In the case of diploid nuclei, the length of DNA is about $2 \mathrm{~m}$. Now, the thickness of the DNA is $20 \AA$ [70]. Approximating DNA as a cylinder, the volume of DNA is about $\pi R^{2} L \sim 3.14 \times\left(400 \times 10^{-20}\right) \times$ $2 \sim 25 \times 10^{-18} \mathrm{~m}^{3}=25 \mu \mathrm{m}^{3}$. As the number of base pairs per nucleosome is about 200 [71], the number of histone octamer cores will be about $\left(3 \times 10^{9}\right) / 200=1.5 \times 10^{7}$. The histone octamer can be approximated by a disc of $110 \times$ $110 \times 57 \AA[57,69,72], 5.41 \times 10^{5} \AA^{3}$. Thus, the volume of all the histones would be $\left(1.5 \times 10^{7}\right) \times(5.41 \times$ $\left.10^{5} \times 10^{-30}\right) \mathrm{m}^{3} \sim 81.15 \times 10^{-19} \mathrm{~m}^{3} \sim 8 \mu \mathrm{m}^{3}$. The total volume of chromatin is $\sim$ volume of DNA + volume of histones octamers + linker proteins (which we ignore for simplicity) $\sim 25+8 \sim 33 \mu \mathrm{m}^{3}$. Considering the nucleus to be of radius $5 \mu \mathrm{m}$, the volume of the nucleus is about
$523 \mu \mathrm{m}^{3}$. Using this value, the volume fraction of the chromatin is about $33 / 523 \sim 0.06$. A reasonable value to consider is then 0.1 , which we have used in our calculations. This calculation is similar to that for Arabidopsis cell nuclei in Ref. [73].

\section{APPENDIX B: EXACT SOLUTION OF THE ANISOTROPIC CASE FOR A HARMONIC EPIGENETIC POTENTIAL IN THE ABSENCE OF NOISE}

Our governing equations represent a three-dimensional, coupled and, in general, nonlinear dynamical system. The choice of a harmonic epigenetic potential $\delta \Psi^{2} / 2$ and a constant force $f$ yields a linear system of equations that can be written as

$$
\begin{aligned}
\delta \dot{\Psi} & =-A \delta \Psi+B\left(\frac{1}{2} \delta R_{\|}+\delta R_{\perp}\right) \\
\delta \dot{R}_{\|} & =-C \delta R_{\|}-D \delta \Psi-f \\
\delta \dot{R}_{\perp} & =-C \delta R_{\perp}-D \delta \Psi
\end{aligned}
$$

We define the Laplace transform and its inverse as

$$
\begin{aligned}
X(s) & =\int_{0}^{\infty} x(t) e^{-s t} d t \\
x(t) & =\frac{1}{2 \pi i} \lim _{T \rightarrow \infty} \int_{\gamma-i T}^{\gamma+i T} X(s) e^{s t} d s,
\end{aligned}
$$

where the integration is done along the vertical line $\operatorname{Re}(s)=\gamma$ in the complex plane so that $\gamma$ is greater than the real part of all singularities of $X(s)$. Using the definition in Eq. (B2), we take the Laplace transform of Eqs. (B1),

$$
\begin{aligned}
s \delta \Psi(s)-\delta \Psi(0) & =-A \delta \Psi(s)+\frac{B}{2} \delta R_{\|}(s)+B \delta R_{\perp}(s), \\
s \delta R_{\|}(s)-\delta R_{\|}((0) & =-C \delta R_{\|}(s)-D \delta \Psi(s)-\frac{f}{s} \\
s \delta R_{\perp}(s)-\delta R_{\perp}(0) & =-C \delta R_{\perp}(s)-D \delta \Psi(s) .
\end{aligned}
$$

For the simplest initial condition, with $\delta \Psi(0)=\delta R_{\|}(0)=$ $\delta R_{\perp}(0)=0$, taking the inverse Laplace transform yields the solution

$$
\begin{aligned}
\delta \Psi(t) & =-\frac{B f}{2}\left(a+b e^{c_{11} t}+c e^{c_{22} t}\right), \\
\delta R_{\|}(t) & =\frac{B D f}{2}\left(a_{1}-b_{1} e^{-C t}+c_{1} e^{c_{11} t}+d_{1} e^{c_{22} t}\right)-f, \\
\delta R_{\perp}(t) & =\frac{B D f}{2}\left(a_{1}-b_{1} e^{-C t}+c_{1} e^{c_{11} t}+d_{1} e^{c_{22} t}\right),
\end{aligned}
$$

where the constants are given by the following, 


$$
\begin{aligned}
a & =\frac{1}{c_{11} c_{22}}, \\
b & =\frac{1}{c_{11}\left(c_{11}-c_{22}\right)}, \\
c & =\frac{1}{c_{22}\left(c_{22}-c_{11}\right)}, \\
a_{1} & =\frac{a}{C}, \\
b_{1} & =\frac{1}{C\left(C+c_{11)}\left(C+c_{22}\right)\right.}, \\
c_{1} & =\frac{b}{\left(C+c_{11}\right)}, \\
d_{1} & =\frac{c}{\left(C+c_{22}\right)},
\end{aligned}
$$

and

$$
c_{11,22}=\frac{-(A+C) \pm \sqrt{(C-A)^{2}-6 B D}}{2} .
$$

At long times, these solutions attain steady-state values that vary linearly with the applied force $f$.

\section{APPENDIX C: RECONSTRUCTION OF THE POTENTIAL LANDSCAPE}

A simpler 2D analog of the dynamical system (1) can be written as follows:

$$
\begin{gathered}
\delta \dot{\Psi}=-\frac{\partial V(\delta \Psi)}{\partial \delta \Psi}+B \delta R+\eta(t), \\
\dot{\delta R}=-C \delta R-D \delta \Psi .
\end{gathered}
$$

Assuming that the dynamics of $\delta R$ is much slower than that of $\delta \Psi$, we can consider $\delta R$ as a constant in the $\delta \dot{\Psi}$ equation. This results in

$$
\delta \Psi=-\frac{\partial}{\partial \delta \Psi}[V(\delta \Psi)-B \delta R \delta \Psi]+\eta(t) .
$$

The corresponding Fokker-Planck equation can be written as

$$
\frac{\partial P(\delta \Psi, t)}{\partial t}=\frac{\partial}{\partial \delta \Psi}\left[\frac{\partial V_{\mathrm{eff}}(\delta \Psi)}{\partial \delta \Psi} P(\delta \Psi, t)\right]+\frac{1}{2} \frac{\partial^{2}}{\partial^{2} \delta \Psi} P(\delta \Psi, t),
$$

where $V_{\text {eff }}(\delta \Psi)=V(\delta \Psi)-B \delta R \delta \Psi$.

For the steady-state solution $\partial P / \partial t=0$,

$$
\frac{\partial}{\partial \delta \Psi}\left[\frac{\partial V_{\mathrm{eff}}(\delta \Psi)}{\partial \delta \Psi} P(\delta \Psi, t)\right]+\frac{1}{2} \frac{\partial^{2}}{\partial^{2} \delta \Psi} P(\delta \Psi, t)=0,
$$

or

$$
\frac{\partial}{\partial \delta \Psi}\left[\frac{\partial V_{\mathrm{eff}}(\delta \Psi)}{\partial \delta \Psi} P_{s}(\delta \Psi)+\frac{1}{2} \frac{\partial}{\partial \delta \Psi} P_{s}(\delta \Psi)\right]=0=\frac{\partial}{\partial \delta \Psi} j(\delta \Psi)
$$

In a steady state, the flux $j(\delta \Psi)$ vanishes; thus,

$$
\left[\frac{d}{d \delta \Psi}+2 \frac{d V_{\mathrm{eff}}(\delta \Psi)}{d \delta \Psi}\right] P_{s}(\delta \Psi)=0
$$

The above equation can be solved for the values of $\delta \Psi$ for a constant value of $\delta R$. Once we have those values, we can obtain the distribution $P(\delta \Psi)$. Taking the negative log of this result yields the effective potential $V_{\text {eff }}$, as

$$
\begin{aligned}
P_{s}(\delta \Psi) & \sim \exp \left(-2 V_{\mathrm{eff}}(\delta \Psi)\right), \\
P_{S}(\delta \Psi) & \sim \exp (-2(V(\delta \Psi))-B \delta R \delta \Psi), \\
V(\delta \Psi) & \sim-0.5 \ln \left[P_{s}(\delta \Psi)\right]+B \delta R \delta \Psi .
\end{aligned}
$$

For the parameter value $B=0$, this expression relates the probability distribution $P(\delta \Psi)$ to the potential landscape $V(\delta \Psi)$. This strategy is used in the reconstruction of the potential landscape in Fig. 6 of the main text.

[1] R. A. Young, Control of the Embryonic Stem Cell State, Cell 144, 940 (2011).

[2] Y. Suda, M. Suzuki, Y. Ikawa, and S. Aizawa, Mouse Embryonic Stem Cells Exhibit Indefinite Proliferative Potential, J. Cell. Physiol. 133, 197 (1987).

[3] S.-J. Heo, B. D. Cosgrove, E. N. Dai, and R. L. Mauck, Mechano-Adaptation of the Stem Cell Nucleus, Nucleus 9, 9 (2018).

[4] S. L. Berger, The Complex Language of Chromatin Regulation During Transcription, Nature (London) 447, 407 (2007).

[5] R. D. Hawkins, G. C. Hon, L. K. Lee, Q. Ngo, R. Lister, M. Pelizzola, L. E. Edsall, S. Kuan, Y. Luu, S. Klugman et al., Distinct Epigenomic Landscapes of Pluripotent and Lineage-Committed Human Cells, Cell Stem Cell 6, 479 (2010).

[6] O. J. Rando and H. Y. Chang, Genome-Wide Views of Chromatin Structure, Annu. Rev. Biochem. 78, 245 (2009).

[7] G. J. Narlikar, H.-Y. Fan, and R. E. Kingston, Cooperation between Complexes that Regulate Chromatin Structure and Transcription, Cell 108, 475 (2002).

[8] S. Efroni, R. Duttagupta, J. Cheng, H. Dehghani, D. J. Hoeppner, C. Dash, D. P. Bazett-Jones, S. Le Grice, R. D. McKay, K.H. Buetow et al., Global Transcription in Pluripotent Embryonic Stem Cells, Cell Stem Cell 2, 437 (2008).

[9] J. G. McNally, Foreword: Biophysics in Chromatin Structure and Nuclear Dynamics, Chromosome Res. 19, 1 (2011). 
[10] E. Meshorer, D. Yellajoshula, E. George, P. J. Scambler, D. T. Brown, and T. Misteli, Hyperdynamic Plasticity of Chromatin Proteins in Pluripotent Embryonic Stem Cells, Dev. Cell 10, 105 (2006).

[11] A. Gaspar-Maia, A. Alajem, E. Meshorer, and M. RamalhoSantos, Open Chromatin in Pluripotency and Reprogramming, Nat. Rev. Mol. Cell Biol. 12, 36 (2011).

[12] A. J. Engler, S. Sen, H. L. Sweeney, and D. E. Discher, Matrix Elasticity Directs Stem Cell Lineage Specification, Cell 126, 677 (2006).

[13] T. Chen and S. Y. Dent, Chromatin Modifiers and Remodellers: Regulators of Cellular Differentiation, Nat. Rev. Genet. 15, 93 (2014).

[14] F. Ugarte, R. Sousae, B. Cinquin, E. W. Martin, J. Krietsch, G. Sanchez, M. Inman, H. Tsang, M. Warr, E. Passegué et al., Progressive Chromatin Condensation and $\mathrm{H} 3 \mathrm{~K} 9$ Methylation Regulate the Differentiation of Embryonic and Hematopoietic Stem Cells, Stem Cell Reports 5, 728 (2015).

[15] R. T. Justinand and A. J. Engler, Stiffness Gradients Mimicking In Vivo Tissue Variation Regulate Mesenchymal Stem Cell Fate, PLoS One 6, e15978 (2011).

[16] N. D. Evans, C. Minelli, E. Gentleman, V. LaPointe, S. N. Patankar, M. Kallivretaki, X. Chen, C. J. Roberts, and M. M. Stevens, Substrate Stiffness Affects Early Differentiation Events in Embryonic Stem Cells, Eur. Cell Matter 18, e13 (2009).

[17] A. Bošković, A. Eid, J. Pontabry, T. Ishiuchi, C. Spiegelhalter, E. V.R. Ram, E. Meshorer, and M.-E. Torres-Padilla, Higher Chromatin Mobility Supports Totipotency and Precedes Pluripotency In Vivo, Genes Dev. 28, 1042 (2014).

[18] D. Dado, M. Sagi, S. Levenberg, and A. Zemel, Mechanical Control of Stem Cell Differentiation, Regener. Med. 7, 101 (2012).

[19] E. K. Yim and M. P. Sheetz, Force-Dependent Cell Signaling in Stem Cell Differentiation, Stem Cell Res. Therapy 3, 41 (2012).

[20] N. S. Hwang, S. Varghese, H. J. Lee, Z. Zhang, and J. Elisseeff, Biomaterials Directed In Vivo Osteogenic Differentiation of Mesenchymal Cells Derived from Human Embryonic Stem Cells, Tissue Eng. Part A 19, 1723 (2013).

[21] C. H. Waddington, Organisers and Genes (Cambridge Biological Studies. University Press, Cambridge, England, 1940).

[22] S. F. Gilbert, Diachronic Biology Meets Evo-Devo: C. H. Waddington's Approach to Evolutionary Developmental Biology, Am. Zool. 40, 729 (2000).

[23] C. H. Waddington, The Strategy of the Genes (Routledge, New York, 2014), Vol. 20.

[24] S. Huang, The Molecular and Mathematical Basis of Waddington's Epigenetic Landscape: A Framework for Post-Darwinian Biology?, BioEssays 34, 149 (2012).

[25] S. A. Kauffman, The Origins of Order: Self-Organization and Selection in Evolution, in Spin Glasses and Biology (World Scientific, Singapore, 1992), pp. 61-100.

[26] J. N. Onuchic, Z. Luthey-Schulten, and P. G. Wolynes, Theory of Protein Folding: The Energy Landscape Perspective, Annu. Rev. Phys. Chem. 48, 545 (1997).
[27] Y. A. Miroshnikova, M. M. Nava, and S. A. Wickström, Emerging Roles of Mechanical Forces in Chromatin Regulation, J. Cell Sci. 130, 2243 (2017).

[28] K. J. Chalut, M. Höpfler, F. Lautenschläger, L. Boyde, C. J. Chan, A. Ekpenyong, A. Martinez-Arias, and J. Guck, Chromatin Decondensation and Nuclear Softening Accompany Nanog Downregulation in Embryonic Stem Cells, Biophys. J. 103, 2060 (2012).

[29] J. Swift and D. E. Discher, The Nuclear Lamina is Mechano-Responsive to ECM Elasticity in Mature Tissue, J. Cell Sci. 127, 3005 (2014).

[30] L. D. Landau and E. Lifshitz, Theory of Elasticity, Vol. 7, Course of Theoretical Physics 3, 109 (1986).

[31] P. M. Chaikin, T. C. Lubensky, and T. A. Witten, Principles of Condensed Matter Physics (Cambridge University Press, Cambridge, England, 1995), Vol. 1.

[32] K. E. Evans and A. Alderson, Auxetic Materials: Functional Materials and Structures from Lateral Thinking, Adv. Mater. 12, 617 (2000).

[33] J. N. Grima, R. Gatt, N. Ravirala, A. Alderson, and K. E. Evans, Negative Poisson's Ratios in Cellular Foam Materials, Mater. Sci. Eng. A 423, 214 (2006).

[34] S. Pagliara, K. Franze, C. R. McClain, G. W. Wylde, C. L. Fisher, R. J. M. Franklin, A. J. Kabla, U. F. Keyser, and K. J. Chalut, Auxetic Nuclei in Embryonic Stem Cells Exiting Pluripotency, Nat. Mater. 13, 638 (2014).

[35] C. Huang and L. Chen, Negative Poisson's Ratio in Modern Functional Materials, Adv. Mater. 28, 8079 (2016).

[36] X. Ren, R. Das, P. Tran, T. D. Ngo, and Y. M. Xie, Auxetic Metamaterials and Structures: A Review, Smart Mater. Struct. 27, 023001 (2018).

[37] A. Alderson, K. L. Alderson, P. J. Davies, and G. M. Smart, The Effects of Processing on the Topology and Mechanical Properties of Negative Poisson's Ratio Foams, in ASME 2005 International Mechanical Engineering Congress and Exposition (American Society of Mechanical Engineers, New York, 2005), pp. 503-510.

[38] J. D. Pajerowski, K. N. Dahl, F. L. Zhong, P. J. Sammak, and D. E. Discher, Physical Plasticity of the Nucleus in Stem Cell Differentiation, Proc. Natl. Acad. Sci. U.S.A. 104, 15619 (2007).

[39] A. G. Larson, D. Elnatan, M. M. Keenen, M. J. Trnka, J. B. Johnston, A. L. Burlingame, D. A. Agard, S. Redding, and G. J. Narlikar, Liquid Droplet Formation by HP1 $\alpha$ Suggests a Role for Phase Separation in Heterochromatin, Nature (London) 547, 236 (2017).

[40] A. R. Strom, A. V. Emelyanov, M. Mir, D. V. Fyodorov, X. Darzacq, and G. H. Karpen, Phase Separation Drives Heterochromatin Domain Formation, Nature (London) 547, 241 (2017).

[41] T. Yamamoto and H. Schiessel, Chromatin Gels Are Auxetic Due to Cooperative Nucleosome Assembly and Disassembly Dynamics, Europhys. Lett. 118, 28003 (2017).

[42] N. Ganai, S. Sengupta, and G. I. Menon, Chromosome Positioning from Activity-Based Segregation, Nucleic Acids Res. 42, 4145 (2014).

[43] A. Agrawal, N. Ganai, S. Sengupta, and G. I. Menon, Chromatin as Active Matter, J. Stat. Mech. (2017) 014001. 
[44] A. Agrawal, N. Ganai, S. Sengupta, and G. I. Menon, A First-Principles Approach to Large-Scale Nuclear Architecture, bioRxiv, 315812 (2018).https://doi.org/10.1101/ 315812

[45] S. Talwar, A. Kumar, M. Rao, G. I. Menon, and G. Shivashankar, Correlated Spatio-Temporal Fluctuations in Chromatin Compaction States Characterize Stem Cells, Biophys. J. 104, 553 (2013).

[46] M. Doi, Introduction to Polymer Physics (Oxford University, New York, 1996).

[47] M. Muthukumar, Polymers under Confinement, in Advances in Chemical Physics (Wiley-Blackwell, New York, 2012), pp. 129-196.

[48] S. Whitaker, Flow in Porous Media I: A Theoretical Derivation of Darcy's Law, Transport Porous Media 1, 3 (1986).

[49] See Supplemental Material at http://link.aps.org/ supplemental/10.1103/PhysRevX.9.041020 for a physical argument for the origins of auxetic behaviour as well as explicit analytic and numerical solutions for our model in specific regimes.

[50] L. I. Davis, The Nuclear Pore Complex, Annu. Rev. Biochem. 64, 865 (1995).

[51] N. Caille, O. Thoumine, Y. Tardy, and J.-J. Meister, Contribution of the Nucleus to the Mechanical Properties of Endothelial Cells, J. Biomech. 35, 177 (2002).

[52] F. Guilak, J. R. Tedrow, and R. Burgkart, Viscoelastic Properties of the Cell Nucleus, Biochem. Biophys. Res. Commun. 269, 781 (2000).

[53] K. N. Dahl, A. J. Engler, J. D. Pajerowski, and D. E. Discher, Power-Law Rheology of Isolated Nuclei with Deformation Mapping of Nuclear Substructures, Biophys. J. 89, 2855 (2005).

[54] A. M. Mastro, M. A. Babich, W. D. Taylor, and A. D. Keith, Diffusion of a Small Molecule in the Cytoplasm of Mammalian Cells, Proc. Natl. Acad. Sci. U.S.A. 81, 3414 (1984).

[55] W. W. Franke, On the Universality of Nuclear Pore Complex Structure, Cell Tissue Res. 105, 405 (1970).

[56] J. Finch, L. Lutter, D. Rhodes, R. Brown, B. Rushton, M. Levitt, and A. Klug, Structure of Nucleosome Core Particles of Chromatin, Nature (London) 269, 29 (1977).

[57] K. Luger, A. W. Mäder, R. K. Richmond, D. F. Sargent, and T. J. Richmond, Crystal Structure of the Nucleosome Core Particle at $2.8 \AA$ Resolution, Nature (London) 389, 251 (1997).

[58] T. J. Richmond and C. A. Davey, The Structure of DNA in the Nucleosome Core, Nature (London) 423, 145 (2003).

[59] J. Heslop-Harrison, A. Leitch, T. Schwarzacher, J. Smith, M. Atkinson, and M. Bennett, The Volumes and Morphology of
Human Chromosomes in Mitotic Reconstructions, Hum. Genet. 84, 27 (1989).

[60] J. Irianto, C. R. Pfeifer, R. R. Bennett, Y. Xia, I. L. Ivanovska, A. J. Liu, R. A. Greenberg, and D. E. Discher, Nuclear Constriction Segregates Mobile Nuclear Proteins Away from Chromatin, Mol. Biol. Cell 27, 4011 (2016).

[61] A. Bancaud, S. Huet, N. Daigle, J. Mozziconacci, J. Beaudouin, and J. Ellenberg, Molecular Crowding Affects Diffusion and Binding of Nuclear Proteins in Heterochromatin and Reveals the Fractal Organization of Chromatin, EMBO J. 28, 3785 (2009).

[62] C. Furusawa and K. Kaneko, A Dynamical-Systems View of Stem Cell Biology, Science 338, 215 (2012).

[63] M. Li, G.-H. Liu, and J. C. I. Belmonte, Navigating the Epigenetic Landscape of Pluripotent Stem Cells, Nat. Rev. Mol. Cell Biol. 13, 524 (2012).

[64] C. M. Rivera and B. Ren, Mapping Human Epigenomes, Cell 155, 39 (2013).

[65] M. Golkaram, J. Jang, S. Hellander, K. S. Kosik, and L. R. Petzold, The Role of Chromatin Density in Cell Population Heterogeneity During Stem Cell Differentiation, Sci. Rep. 7, 13307 (2017).

[66] X. Mao, N. Gavara, and G. Song, Nuclear Mechanics and Stem Cell Differentiation, Stem Cell Rev. Rep. 11, 804 (2015).

[67] E. Meshorer and T. Misteli, Chromatin in Pluripotent Embryonic Stem Cells and Differentiation, Nat. Rev. Mol. Cell Biol. 7, 540 (2006).

[68] J. Swift, I. L. Ivanovska, A. Buxboim, T. Harada, P. D. P. Dingal, J. Pinter, J. D. Pajerowski, K. R. Spinler, J.-W. Shin, M. Tewari et al., Nuclear Lamin-A Scales with Tissue Stiffness and Enhances Matrix-Directed Differentiation, Science 341, 1240104 (2013).

[69] J. Finch, R. Brown, D. Rhodes, T. Richmond, B. Rushton, L. Lutter, and A. Klug, X-Ray Diffraction Study of a New Crystal Form of the Nucleosome Core Showing Higher Resolution, J. Mol. Biol. 145, 757 (1981).

[70] H. Schiessel, The Physics of Chromatin, J. Phys. Condens. Matter 15, R699 (2003).

[71] J. D. McGhee and G. Felsenfeld, Nucleosome Structure, Annu. Rev. Biochem. 49, 1115 (1980).

[72] B.-C. Wang, J. Rose, G. Arents, and E. Moudrianakis, The Octameric Histone Core of the Nucleosome: Structural Issues Resolved, J. Mol. Biol. 236, 179 (1994).

[73] S. De Nooijer, J. Wellink, B. Mulder, and T. Bisseling, Nonspecific Interactions Are Sufficient to Explain the Position of Heterochromatic Chromocenters and Nucleoli in Interphase Nuclei, Nucleic Acids Res. 37, 3558 (2009). 